\title{
Gelfand spectra in Grothendieck toposes using geometric mathematics
}

\author{
Bas Spitters \\ VALS-LRI, Université Paris-Sud/INRIA Saclay \\ bas.spitters@inria.fr \\ Steven Vickers * \\ School of Computer Science, University of Birmingham, \\ Birmingham, B15 2TT, UK. \\ s.j.vickers@cs.bham.ac.uk \\ Sander Wolters ${ }^{\dagger}$ \\ Radboud University Nijmegen, IMAPP \\ s.wolters@math.ru.nl
}

\begin{abstract}
In the (covariant) topos approach to quantum theory by Heunen, Landsman and Spitters, one associates to each unital $\mathrm{C}^{*}$-algebra $A$ a topos $\mathscr{T}(A)$ of sheaves on a locale and a commutative $\mathrm{C}^{*}$-algebra $\underline{A}$ within that topos. The Gelfand spectrum of $\underline{A}$ is a locale $\underline{\Sigma}$ in this topos, which is equivalent to a bundle over the base locale. We further develop this external presentation of the locale $\underline{\Sigma}$, by noting that the construction of the Gelfand spectrum in a general topos can be described using geometric logic. As a consequence, the spectrum, seen as a bundle, is computed fibrewise.

As a by-product of the geometricity of Gelfand spectra, we find an explicit external description of the spectrum whenever the topos is a functor category. As an intermediate result we show that locally perfect maps compose, so that the externalization of a locally compact locale in a topos of sheaves over a locally compact locale is locally compact, too.
\end{abstract}

\section{Introduction}

The main subject of this paper is the interplay between geometric logic and topos-theoretic approaches to $\mathrm{C}^{*}$-algebras (motivated by quantum theory). In particular, we consider the approach of Heunen, Landsman and Spitters [8, 21, 23, 22], although some of the ideas and techniques in this paper may turn out to be of interest to the related approach by Butterfield, Isham and Döring [25, 6, 7, 20, 15] as well; see [50] for a comparison. We are mainly interested in the spectral object of the topos approach and its construction using geometric logic.

We assume that the reader is familiar with the basics of topos and locale theory 1 All toposes are assumed to be Grothendieck toposes, and, in particular, every topos has a natural numbers object (NNO). In its general form, the theory of Grothendieck toposes is that of bounded toposes over some base topos $\mathscr{S}$ that embodies the ambient logic. We shall rarely need to be explicit about $\mathscr{S}$, but our techniques will be valid for $\mathscr{S}$ an arbitrary elementary topos with NNO and thus have wide constructive applicability.

\footnotetext{
*Supported by the UK Engineering and Physical Sciences Research Council, on the project EP/G046298/1 "Applications of geometric logic to topos approaches to quantum theory".

†Supported by N.W.O. through project 613.000.811.

${ }^{1}$ The standard reference [33] contains much of the needed material. In particular, Chapter II of this book demonstrates how sheaves on a topological space can be seen as bundles. Chapter III gives an introduction to Grothendieck toposes. Section VII.1 contains background information on geometric morphisms. Finally Chapter IX gives all background information on locales. At certain points in this paper, in particular in Subsection 3.4 sheaf semantics is used. The relevant background material can be found in Chapter VI of [33]. Another standard reference is the massive work [30, 31].
} 
The paper is split into two sections. Section 2 gives background information on geometric logic and, more importantly, the practical impact of the geometric mathematics that develops from it. For this we can give a first definition as topos-valid constructions on sets (understood as objects of a topos) which are preserved by inverse image functors of geometric morphisms. This is the geometric mathematics of sets, as objects of a topos.

More profoundly, we can also consider geometricity of topos-valid constructions on locales, and much of our ability to do this follows from two results in [32]. The first provides a localic version of a well known result from point-set topology, that sheaves over $X$ are equivalent to local homeomorphisms with codomain $X$. On the one hand, the sheaves are the "sets" in the topos $\operatorname{Sh}(X)$ of sheaves, or the discrete locales - the frames are the powerobjects. On the other hand, the local homeomorphisms can be understood as the fibrewise discrete bundles over $X$, where we understand "bundle" in the very general sense of locale map with codomain $X$. Applying an inverse image functor $f^{*}$ to the sheaf corresponds to pulling back the bundle along $f$. Hence geometricity of a construction on the sheaves corresponds to preservation under pullback of the corresponding construction on the bundles.

The second result in [32] is that internal frames in $\operatorname{Sh}(X)$ are dual to localic bundles over $X$. This immediately allows us to extend our definition of geometricity to constructions on locales, namely as preservation under pullback of the bundle constructions. Since the fibres are got as pullbacks along points, the geometric mathematics works fibrewise and provides a fibrewise topology of bundles. This idea has already been explored in an ad hoc fashion in point-set topology - see, e.g., [26] -, and the notion of geometricity makes it much more systematic when one combines point-free topology with toposes.

It should be noted that pullback of a bundle along a map $f$ is not achieved by applying $f^{*}$ to the internal frame. This is already clear in the fibrewise discrete (local homeomorphism) case, since $f^{*}$ does not preserve powerobjects, nor frame structure in general. [32] define a different functor $f^{\#}$ that transforms frames to frames, but in practice it is often convenient to bypass the frames altogether and instead use presentations of them by generators and relations. [41] shows that applying $f^{*}$ to the presentation corresponds to pullback of the bundle, even though the middle step of presenting a frame is not geometric.

The practical effect of switching to generators and relations is that a locale is described by means of a geometric theory whose models are the (generalized) points of the locale. Hence we explicitly describe the points rather than the opens, though the nature of geometric theories ensures that the topology is described implicitly at the same time, by presenting a frame. Geometricity has the effect of restoring the points to point-free topology, and allows us to define maps $f: Y \rightarrow X$ in two very intuitive ways as geometric transformations: as a map, $f$ transforms points of $Y$ to points of $X$, while as a bundle it transforms points of $X$ to locales, the fibres, and defines the bundle locale $Y$ at the same time. This is explained in more detail in [48].

This switch of emphasis can be disconcerting if one thinks that a locale $i s$ its frame. However, calculating an internal frame is error prone - experience shows this even in the simpler case of presheaf toposes -, and one of the central messages of this paper is that the geometric methods often allow us to avoid the frame. On the other hand, sometimes the frame is still needed. Later on we show how to exploit local compactness to give a geometric calculation of it. Specifically, we prove a result about exponentiability of objects in a topos, which entails that if $X$ is a locally compact locale and $\underline{Y}$ is a locally compact locale in $\operatorname{Sh}(X)$, with bundle locale $Y$ over $X$, then $Y$ is locally compact.

Section 3 applies the previous discussion about geometricity to the HLS topos approach of quantum theory. Since the topos is that of sheaves over a locale, the spectrum can be understood as a bundle [16], in which each fibre is the spectrum of a commutative $C^{*}$-algebra, and the geometric approach will emphasize this. In particular, we concentrate on the external description of the bundle locale, which is 
the phase space of the topos approach. This phase space is the Gelfand spectrum of a unital commutative $\mathrm{C}^{*}$-algebra, internal to a functor category. The first two subsections seek a fully geometric description of the construction of this spectrum. The basic problem is that, geometrically, a $\mathrm{C}^{*}$-algebra has a natural topology and therefore should be a locale rather than a set. As far as we know, there is not yet a satisfactory account of Gelfand duality that deals with the $C^{*}$-algebra in this way. [4] defines $C^{*}$-algebra in a way that is topos-valid but not geometric - it uses a non-geometric characterization of completeness. We show how to replace the complete $\mathrm{C}^{*}$-algebra by a geometric stucture that yields the same spectrum, and following [9] we quickly reduce to the spectrum of a normal distributive lattice, a general lattice theoretic way to present compact regular locales. In the subsection that follows these ideas are used to find an explicit description of the phase space in question. This is subsequently generalized to a description of Gelfand spectra of arbitrary unital commutative $\mathrm{C}^{*}$-algebras in functor categories. In the final subsection we briefly consider generalizing the topos approach to $\mathrm{C}^{*}$-algebras to algebraic quantum field theory.

\section{Geometricity}

This section is divided into four parts. In Subsection 2.1 we briefly discuss geometric logic and associated mathematical constructions. An important point is that the constructions that can be expressed by geometric mathematics coincide with the constructions that are preserved when pulled back along the inverse image functor of a geometric morphism. Subsection 2.2 shifts attention from geometric logic to geometric mathematics (in particular by using geometric type constructs). We discuss geometricity of topos-valid constructions on sets (in the generalized sense of objects in a topos) and on locales. Subsequently, in Subsection 2.3 the geometricity of such constructions is further analysed when these constructions are carried out in toposes that are functor categories and in toposes of sheaves on a topological space. Finally, in Subsection 2.4 we discuss exponentiability of locales.

The results of this section are important in two ways. First, they allow us to talk about the opens, the elements of a frame (which is itself not a geometric concept), in a geometric way. Second, it entails that the external description of the Gelfand spectrum investigated in the next section is a locally compact locale.

\subsection{Geometric logic}

We briefly describe geometric theories and their interpretations in toposes. The discussion is brief because the work in Section 3 concentrates on geometric mathematics, as described in Subsection 2.2 , rather than geometric logic as presented in this subsection. The reader is advised to browse rather than carefully read this subsection, and to consult it at a later stage, if needed.

In this subsection we follow the discussion in [30, Section D1] where a much more detailed presentation is given. Section 3 of [45] also treats this material, with more emphasis on geometric logic. Another good source is [33, Chapter X], but note that what is called geometric there is called coherent in [30, 31] and [45]: the difference is that "coherent" forbids infinite disjunctions. Finally, [48] provides an alternative introduction to some of the techniques presented in this section.

The language of geometric logic is that of an infinitary, first-order, many-sorted predicate logic with equality 2. We start with a first-order signature $\Sigma$ (where the notation $\Sigma$ has nothing to do with the spectrum $\Sigma$ in the following sections). The signature consists of a set $S$ of sorts, a set $F$ of function symbols and a set $R$ of predicate symbols. Each function symbol $f \in F$ has a type, which is a non-empty

\footnotetext{
${ }^{2}$ We shall not explicitly consider the geometric types of e.g. [45] Subsection 3.4].
} 
finite list of sorts $A_{1}, \ldots, A_{n}, B$. We write this as $f: A_{1} \times \ldots \times A_{n} \rightarrow B$, in anticipation of the categorical interpretation of the function symbols. The number $n$ is called the arity of the function symbol. If the arity of a function symbol $f$ is 0 , i.e., when its type is a single sort $B$ (we will write $f: 1 \rightarrow B$ ), $f$ is called a constant. Each predicate $P \in R$ also has a type $A_{1}, \ldots, A_{n}$, where we also allow the empty list $(n=0)$ as a type. We will write $P \subseteq A_{1} \times \ldots \times A_{n}$, again in anticipation of the categorical representation. A predicate with arity equal to 0 , written as $P \subseteq 1$, will be called a propositional symbol.

Given the signature $\Sigma$, a contex $\mathrm{I}^{3}$ is a finite set of variables, each associated with some sort. It is customary to write the set as a vector $\vec{x}=\left(x_{1}, \ldots, x_{n}\right)$ of distinct variables. Using $\Sigma$ and the variables we can define terms and formulae over $\Sigma$ in that context. Terms, which all have a sort assigned to them, are inductively defined as follows:

- Each variable $x$ of sort $A$ is a term of sort $A$.

- If $f: A_{1} \times \ldots \times A_{n} \rightarrow B$ is a function symbol, and $t_{1}, \ldots, t_{n}$ are terms of sort $A_{1}, \ldots, A_{n}$, then $f\left(t_{1}, \ldots, t_{n}\right)$ is a term of sort $B$. In particular, every constant is a term.

We can now construct geometric formulae in that context inductively as follows. They form the smallest class which is closed under the following clauses:

1. If $R \subseteq A_{1} \times \ldots \times A_{n}$ is a predicate, and $t_{1}, \ldots, t_{n}$ are terms of sort $A_{1}, \ldots, A_{n}$, then $R\left(t_{1}, \ldots t_{n}\right)$ is a formula. For the particular case $n=0$, even in the empty context every propositional symbol is a formula.

2. If $s$ and $t$ are terms of the same sort, then $s=t$ is a formula.

3. Truth $\top$ is a formula. If $\phi$ and $\psi$ are formulae, then so is the conjunction $\phi \wedge \psi$.

4. Let $I$ be any (index) set and for every $i \in I$, let $\phi_{i}$ be a formula. Then $\bigvee_{i \in I} \phi_{i}$ is a formula.

5. If the variable $u$ is not in the context $\vec{x}$, and $\phi$ is a formula in context $\vec{x} \cup\{u\}$, then $(\exists u) \phi$ is a formula in context $\vec{x}$.

Note that implication, negation and the universal quantifier are not allowed as connectives in the construction of geometric formulae.

To show the context explicitly we shall denote a formula or term in context by $\vec{x} . \phi$ or $\vec{x}$.t. Note that a formula or term does not have to use all the variables in its context - some may be unused.

We will also consider sequents $\phi \vdash_{\vec{x}} \psi$, where $\phi$ and $\psi$ are geometric formulae in context $\vec{x}$. We will think of the sequent as expressing that $\psi$ is a logical consequence of $\phi$ in context $\vec{x}$. A geometric theory $\mathbb{T}$ over $\Sigma$ is simply a set of such sequents $\phi \vdash_{\vec{x}} \psi$.

The next step is to consider interpretations of geometric theories in toposes. The discussion will be very brief, but the details can be found in the references stated at the beginning of this subsection. Let $\mathscr{E}$ be a topos (actually any category that has finite products would suffice for defining the structures, and also assuming pullbacks makes the definition of a homomorphism of $\Sigma$-structures nicer). Given a signature $\Sigma$, a $\Sigma$-structure $M$ in $\mathscr{E}$ is defined as follows. For every sort $A$ in $\Sigma$ there is an associated object $M A$ in $\mathscr{E}$. For every function symbol $f: A_{1} \times \ldots A_{n} \rightarrow B$ there is an arrow $M f: M A_{1} \times \ldots \times M A_{n} \rightarrow M B$ in $\mathscr{E}$. A constant $c: 1 \rightarrow B$ is interpreted as an arrow $M c: 1 \rightarrow M B$, where 1 denotes the terminal object of $\mathscr{E}$. A predicate $R$ of type $A_{1} \times \ldots \times A_{n}$ is interpreted as a monic arrow $M R \longmapsto M A_{1} \times \ldots \times M A_{n}$.

If $M$ and $N$ are $\Sigma$-structures in $\mathscr{E}$, then a homomorphism of $\Sigma$-structures $h$ is defined as follows. For each sort $A$ in $\Sigma$ there is an arrow $h_{A}: M A \rightarrow N A$. For each function symbol $f: A_{1} \times \ldots \times A_{n} \rightarrow B$, we demand $h_{B} \circ M f=N f \circ\left(h_{A_{1}} \times \ldots \times h_{A_{n}}\right)$. If $R \subseteq A_{1} \times \ldots \times A_{n}$ is a predicate, then we demand that

\footnotetext{
${ }^{3}$ This should not be confused with the non-logical notion of context used in the topos approaches to quantum theory.
} 
$M R \subseteq\left(h_{A_{1}} \times \ldots \times h_{A_{n}}\right)^{*}(N R)$ holds as subobjects of $M A_{1} \times \ldots \times M A_{n}$, where the right hand side means pulling the monic arrow $N R \longmapsto N A_{1} \times \ldots \times N A_{n}$ back along $h_{A_{1}} \times \ldots \times h_{A_{n}}$.

The $\Sigma$-structures in a topos $\mathscr{E}$ and their homomorphisms define a category $\operatorname{Str}_{\Sigma}(\mathscr{E})$. Let $F: \mathscr{E} \rightarrow \mathscr{F}$ be a functor between toposes. $F$ need not come from a geometric morphism, but we do assume it to be left exact. Any such functor induces a functor $\operatorname{Str}_{\Sigma}(F): \operatorname{Str}_{\Sigma}(\mathscr{E}) \rightarrow \operatorname{Str}_{\Sigma}(\mathscr{F})$ in a straightforward way.

The next step is to introduce models of a geometric theory $\mathbb{T}$ over $\Sigma$. In order to do this we need to interpret terms and formulae-in-context for a $\Sigma$-structure in $\mathscr{E}$. This can be done inductively, in much the same way as in using the internal language of the topos. Details can be found in [30, Subsection D1.2]. For a given $\Sigma$-structure $M$, the end result is that a formula-in-context $\vec{x}$. $\phi$, where $\vec{x}=\left(x_{1}, \ldots, x_{n}\right)$ are variables with associated sorts $A_{1}, \ldots, A_{n}$, is interpreted as a subobject $M(\vec{x} . \phi)$ of $M A_{1} \times \ldots \times M A_{n}$. A $\Sigma$-structure $M$ in a topos $\mathscr{E}$ is called a model for a geometric theory $\mathbb{T}$ if for every sequent $\phi \vdash_{\vec{x}} \psi$ in $\mathbb{T}$ we have $M(\vec{x} . \phi) \subseteq M(\vec{x} . \psi)$, where we view the interpretation of the formulae as subobjects of $M A_{1} \times \ldots \times M A_{n}$. We write $\operatorname{Mod}_{\mathbb{T}}(\mathscr{E})$ for the full subcategory of $\operatorname{Str}_{\Sigma}(\mathscr{E})$ whose objects are the models of $\mathbb{T}$.

Although any left-exact functor $F: \mathscr{E} \rightarrow \mathscr{F}$ induces a functor $\operatorname{Str}_{\Sigma}(F)$ between the associated categories of $\Sigma$-structures, in general $F$ does not preserve those "geometric constructions" used to interpret formulae, and consequently $\operatorname{Str}_{\Sigma}(F)$ does not restrict to model categories. On the other hand, if $F=f^{*}$, the inverse image of a geometric morphism $f$, then $\operatorname{Str}_{\Sigma}(F)$ does restrict to a functor $\operatorname{Mod}_{\mathbb{T}}(F)$ : $\operatorname{Mod}_{\mathbb{T}}(\mathscr{E}) \rightarrow \operatorname{Mod}_{\mathbb{T}}(\mathscr{F})$. A nice proof of the restriction is given in [33, Section X.3]. Although only finite joins are considered there, this proof is in particular interesting because it also treats (non-geometric) formulae that use implication and the universal quantifier, and shows that models of a theory using this additional structure are only preserved by the inverse image functor of a geometric morphism when the geometric morphism is open. In general, we shall describe a topos-valid construction as geometric if it is preserved (up to isomorphism) by inverse image functors.

One important kind of mathematical structure that can be expressed by geometric logic is finitary algebraic theories, such as monoids, (Abelian) groups, rings and lattices. Their axioms all take the shape $\top \vdash_{\vec{x}} s=t$ where $s$ and $t$ are terms. These theories are clearly geometric, as are many-sorted algebraic theories such as pairs of rings and modules. The theory of partially ordered sets, but also ordered groups or ordered rings, is geometric. More general examples are local rings, finite sets and (small) categories. See [30, Example D1.1.7] for more details.

Sometimes it can be hard to see whether some given structure is geometric. Just because it is presented by a theory that uses $\forall$ or $\Rightarrow$ in formulae, does not mean that there is no equivalent geometric theory that describes the structure.

One example of structure that is definitely not geometric is that of frames: complete lattices with binary meet distributing over arbitrary joins, and homomorphisms preserving joins and finite meets. This will be important in Section 3, where we will look at spectra of commutative unital $\mathrm{C}^{*}$-algebras in toposes: the spectrum is described by a frame. The non-geometricity of frames is shown by the fact that the inverse image functor of a geometric morphism does not necessarily map frames to frames. $\mathrm{C}^{*}$-algebras provide another example: inverse image functors need not map $\mathrm{C}^{*}$-algebras to $\mathrm{C}^{*}$-algebras. Still, we can say a lot about the spectrum of a commutative $C^{*}$-algebra by paying attention to geometric constructions, as we will see in Subsection 3.2 .

Although there is not a single geometric theory whose models are the frames, they are closely related to the important class of propositional geometric theories - those with no declared sorts so that the signature consists entirely of propositional symbols. Each such theory can be straightforwardly translated into a presentation of a frame by generators and relations, with propositional symbols and axioms becoming the generators and relations, and then the points of the corresponding locale are equivalent to the models 
of the theory.

Defining the notion of geometric theory and their models in toposes is just the start of an introduction to geometric logic. The next step would be to discuss the rules of inference. However, for the purposes of this paper we have treated enough and the reader interested in the rules of inference is directed to [30, Section D1.3].

More important for us will be the way that geometric theories are used to describe the generalized points of a Grothendieck topos $\mathscr{F}$, the geometric morphisms $\mathscr{E} \rightarrow \mathscr{F}$ to $\mathscr{F}$ from another topos. Whenever we refer to points we mean generalized points in the above sense, and not the smaller class of global points, i.e., points where $\mathscr{E}=$ Set. A key fact is that for each $\mathscr{F}$ there is a geometric theory $\mathbb{T}$ such that the points of $\mathscr{F}$ are equivalent to the models of $\mathbb{T}$ in $\mathscr{E}$. $\mathscr{F}$ is said to classify the theory $\mathbb{T}$. Conversely, every geometric theory has a classifying topos.

The same techniques can also be applied to localic toposes, i.e., those of the form $\operatorname{Sh} X$, where $X$ is a locale. They classify propositional geometric theories.

The geometric approach will continually ask - What are the points? What theory does it classify? This will come as a shock to those used to thinking of the points as insufficient - as, indeed, the global points are - and calculating concretely with the topos or the frame.

\subsection{Geometric mathematics}

In Subsection 2.1 we gave formal definitions of geometric theories and their interpretation in toposes. In what follows, however, we shall make little use of formal geometric theories. This is not just for the usual reason, that in practical mathematics it is tedious to work formally. We shall also be extensively using type-theoretic features of the logic that have not been fully formalized. Our informal treatment will be what we describe as "geometric mathematics".

It has two levels. A geometric mathematics of "sets" (understood as objects of a topos) consists of those structures, constructions and theorems that are preserved by inverse image parts of geometric morphisms. The next level is a geometric mathematics of locales, and we shall explain geometricity here in terms of bundles.

A key property of geometric logic, distinguishing it from finitary first-order logics, is that the infinitary disjunctions allow us to characterize some constructions (necessarily geometric) uniquely up to isomorphism by using geometric structure and axioms. These constructions include $\mathbb{N}, \mathbb{Z}$ and $\mathbb{Q}$ (though not $\mathbb{R}$ and $\mathbb{C}$ ), free algebras more generally, colimits and finite limits. Using these constructions informally, but in the knowledge that they could be formalized within geometric theories, allows us to deal with geometric theories and their models in a way that is closer to a "geometric mathematics" than to logic. We shall do this extensively in the rest of the paper.

To explain how this extends to constructions on locales, we shall need to recall the equivalence between internal locales and bundles. For simplicity we assume we are working in a topos $\mathscr{E}=\operatorname{Sh}(X)$, where $X$ is a locale in Set ${ }^{4}$ though the idea works more generally. Then the category $\mathbf{L o c}_{\mathrm{Sh}(X)}$ of locales in $\operatorname{Sh}(X)$ (defined as the dual of the category of internal frames) is equivalent to the slice category Loc $/ X$, of locales in Set over $X$ ([32] or [30, Section C1.6]). A locale $\underline{Y}$ in the topos $\mathscr{E}=\operatorname{Sh}(X)$ can be represented by a locale map $p: Y \rightarrow X$. We will call a map a bundle when we view it in this way, and think of it as a family of locales, the fibres, parametrized by (generalized) points of $X$. Let $x: X^{\prime} \rightarrow X$ be

${ }^{4}$ - or an arbitrary base topos $\mathscr{S}$, elementary with NNO. We do not assume classical sets. 
a point of $X$, and consider the pullback diagram

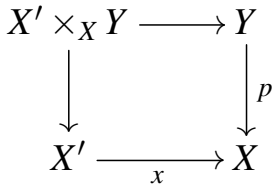

If $X^{\prime}=1$, so $x$ is an ordinary (global) point of $X$, then the pullback $X^{\prime} \times_{X} Y$ is the fibre of $p$ over $x$. When we generalize to arbitrary $X^{\prime}$ then we may think of the pullback as the generalized fibre of $p$ over the generalized point $x$.

If we define toposes $\mathscr{E}=\operatorname{Sh}(X)$ and $\mathscr{F}=\operatorname{Sh}\left(X^{\prime}\right)$, then $x$ becomes a geometric morphism $x: \mathscr{F} \rightarrow$ $\mathscr{E}$. As we have noted earlier, the inverse image part $x^{*}: \mathscr{E} \rightarrow \mathscr{F}$ does not preserve frame structure. Nonetheless, there is a functor $x^{\#}: \mathbf{F r m}_{\mathscr{E}} \rightarrow \mathbf{F r m}_{\mathscr{F}}$ whose action on the corresponding bundles is by pullback along $x$. Moreover it is a right adjoint to $x_{*}: \mathbf{F r m}_{\mathscr{F}} \rightarrow \mathbf{F r m}_{\mathscr{E}}$, the direct image part of $x$, which, unlike $x^{*}$, does preserve frames.

For each object $U$ in a topos $\mathscr{E}$ there is a corresponding discrete locale whose frame is the powerobject $\mathscr{P} U$. The corresponding bundles are the étale bundles, or local homeomorphisms ([33, Chapter II]), whose fibres are normally called stalks. If $F$ is a geometric morphism with codomain $\mathscr{E}$ then $f^{\#}(\mathscr{P} U) \cong \mathscr{P} f^{*}(U)$, and it follows that applying $f^{*}$ corresponds to pulling back the bundle of the discrete locale. Our notion of geometricity with regard to constructions on objects of toposes consisted of preservation under inverse image functors. If we translate the construction into one on the bundles, we see that geometricity comes to mean preservation under pullback.

In this form we have a notion of geometricity that can also be understood with regard to locales, once they are interpreted as bundles. Another way to say the same thing is that the mathematics works fibrewise (for generalized fibres), since fibres are just pullbacks of bundles.

We often make use of the following observation. Let $p: Y \rightarrow X$ be an arbitrary localic bundle: we take it that there is an internal locale in $\operatorname{Sh} X$ corresponding to it. If $y: W \rightarrow Y$ is a (generalized) point of $Y$, then we obtain a point $x=p y$ of $\mathrm{X}$ and a map $y^{\prime}: W \rightarrow x^{*} Y$ to the pullback $x^{*} Y$ :

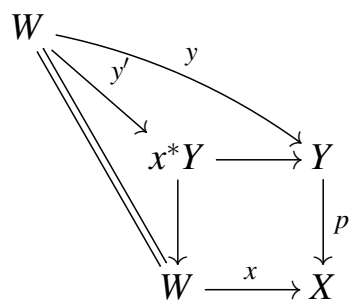

Thus we may describe the points of $Y$ as the pairs $\left(x, y^{\prime}\right)$, where $x$ is a point of $X$ and $y^{\prime}$ is a point of the fibre $x^{*} Y$.

This description is geometric, because the structure and properties of the diagram are preserved by any change of base (pullback along maps into $W$ ). It follows that, for a geometric theory for $Y$, we need one whose models are the points $\left(x, y^{\prime}\right)$.

We shall also need to consider the specialization order $\sqsubseteq$ on $Y$, and for this the analysis needs to be more refined. The straightforward part is that within a single fibre $x^{*} Y$ we have $y_{1}^{\prime} \sqsubseteq y_{2}^{\prime}$ iff $\left(x, y_{1}^{\prime}\right) \sqsubseteq\left(x, y_{2}^{\prime}\right)$. The $\Rightarrow$ direction follows immediately from the map $x^{*} Y \rightarrow Y$, while the converse follows from the fact that in Loc the pullback property determines the hom-posets into a fibre, as well as the homsets. Also, if $\left(x_{1}, y_{1}^{\prime}\right) \sqsubseteq\left(x_{2}, y_{2}^{\prime}\right)$ then $x_{1} \sqsubseteq x_{2}$ by the map $p$. 
For a more precise analysis of the specialization between fibres we shall assume that $p$ is an opfibration in the 2-categorical sense. (A similar analysis applies when $p$ is a fibration. ${ }^{5}$ For a definition, see [30, B4.4.1]. However, easier to understand in our situation is to define the opfibrational structure generically, as in [16], which also discusses other ways in which fibrations and opfibrations relate to the topos approaches to quantum theory. Consider the two maps $X^{i}: X^{\mathbb{S}} \rightarrow X$ where $i$ is either of the two principal points $\perp, \top$ of $\mathbb{S}$. We have obvious maps $Y^{\mathbb{S}} \rightarrow\left(X^{i}\right)^{*} Y$, defined on $\left(x_{\perp}, y_{\perp}^{\prime}, x_{\top}, y_{\top}^{\prime}\right)$ by forgetting one of the $y_{i}^{\prime}$ s. Then $p$ is an opfibration if the map $Y^{\mathbb{S}} \rightarrow\left(X^{\perp}\right)^{*} Y$ has a left adjoint over $\left(X^{\perp}\right)^{*} Y$. We can write it in the form

$$
\left(x_{\perp}, x_{\top}, y_{\perp}^{\prime}\right) \mapsto\left(x_{\perp}, y_{\perp}^{\prime}, x_{\top}, r_{x_{\perp} x_{\top}}\left(y_{\perp}^{\prime}\right)\right),
$$

thus focusing attention on the fibre map $r_{x_{\perp} x_{\top}}: x_{\perp}^{*} Y \rightarrow x_{\top}^{*} Y$.

Proposition 1. Let $p: Y \rightarrow X$ be a locale map that is a 2-categorical opfibration. Then $\left(x_{1}, y_{1}^{\prime}\right) \sqsubseteq\left(x_{2}, y_{2}^{\prime}\right)$ in $Y$ iff $x_{1} \sqsubseteq x_{2}$ in $X$ and $r_{x_{1} x_{2}}\left(y_{1}^{\prime}\right) \sqsubseteq y_{2}^{\prime}$ in $x_{2}^{*} Y$.

Proof. $\Rightarrow$ : We have already noted that $x_{1} \sqsubseteq x_{2}$. Using the adjunction we find $\left(x_{1}, y_{1}^{\prime}, x_{2}, r_{x_{1} x_{2}}\left(y_{1}^{\prime}\right)\right) \sqsubseteq$ $\left(x_{1}, y_{1}^{\prime}, x_{2}, y_{2}^{\prime}\right)$ and the conclusion follows.

$\Leftarrow$ : Using the fact that the adjoint maps into $Y^{\mathbb{S}}$, we know $\left(x_{1}, y_{1}^{\prime}\right) \sqsubseteq\left(x_{2}, r_{x_{1} x_{2}}\left(y_{1}^{\prime}\right)\right) \sqsubseteq\left(x_{2}, y_{2}^{\prime}\right)$.

There is a dual result if $p$ is a fibration, giving fibre maps contravariantly $r_{x_{\top} x_{\perp}}: x_{\top}^{*} Y \rightarrow x_{\perp}^{*} Y$. Then $\left(x_{1}, y_{1}^{\prime}\right) \sqsubseteq\left(x_{2}, y_{2}^{\prime}\right)$ in $Y$ iff $x_{1} \sqsubseteq x_{2}$ in $X$ and $y_{1}^{\prime} \sqsubseteq r_{x_{2} x_{1}}\left(y_{2}^{\prime}\right)$ in $x_{1}^{*} Y$.

If a locale property or construction is understood in terms of frames, then its geometricity itself is likely to be non-obvious, because frames are non-geometric. One potential way round this is to check preservation by the functors $f^{\#}$, but this can be difficult without concrete knowledge of $f^{\#}$. A more practical approach is usually to work with frame presentations by generators and relations. A frame $\mathscr{O} X$ that is presented by a set of generators $G$ and a set of relations $R$ will be denoted as $\mathscr{O} X \cong \mathbf{F r}\langle G \mid R\rangle$. For the reader unfamiliar with frame presentations, see e.g. [39, Chapter 4] for an introduction. A general but primitive class of frame presentations is that of GRD-systems [41, Section 5], but there are various more restricted kinds that are better adapted to particular problems. Of whatever kind, the central property is that the relation between the presentation and the corresponding bundle is geometric - that is, preserved by bundle pullback.

\subsection{Toposes of presheaves and of sheaves}

For the topos approach(es) to quantum theory we are in particular interested in toposes that are either functor categories or sheaves on a locale. In this subsection we consider geometricity for constructions on sets and locales in such toposes. As before, we are considering topos-valid constructions, so sets and their elements are understood in their generalized sense.

For functor categories, the fact that geometric constructions are preserved under the inverse image functor of any geometric morphism entails the following lemma.

Lemma 2. ([30. Corollary D1.2.14(i)]) Let $\mathbb{T}$ be a geometric theory over a signature $\Sigma$ and let $\mathscr{C}$ be any small category. A $\Sigma$-structure $M$ in the topos $[\mathscr{C}$, Set $]$ is a $\mathbb{T}$-model iff for every object $C \in \mathscr{C}_{0}$ the $\Sigma$-structure ev ${ }_{C}(M)$ is a $\mathbb{T}$-model in Set. Here ev $v_{C}:[\mathscr{C}$, Set $] \rightarrow$ Set denotes the functor that evaluates at the object $C$. There is an isomorphism

$$
\operatorname{Mod}_{\mathbb{T}}([\mathscr{C}, \operatorname{Set}]) \cong\left[\mathscr{C}, \operatorname{Mod}_{\mathbb{T}}(\operatorname{Set})\right] .
$$

\footnotetext{
${ }^{5}$ There are also topological notions of fibration, and they are different from the one we use.
} 
In this lemma the "only if" part follows from the fact that $e v_{C}$ is the inverse image part of a geometric morphism. The observation that we have an isomorphism of categories of models uses the fact that a homomorphism of $\Sigma$-structures in $[\mathscr{C}$, Set $]$ can be identified with a natural transformation between the $\Sigma$-structures, viewed as functors $\mathscr{C} \rightarrow \operatorname{Str}_{\Sigma}(\mathbf{S e t})$.

[30. Corollary D1.2.14(ii)] shows that for a spatial locale $X$, it suffices to check the fibrewise nature for fibres over the global points. Let $\mathbb{T}$ be a geometric theory over a signature $\Sigma$. Then a $\Sigma$-structure $M$ in the topos $\operatorname{Sh}(X)$ is a $\mathbb{T}$-model iff, for each global point $x$, the fibre $x^{*}(M)$ is a $\mathbb{T}$-model in Set. Unfortunately, from a geometric point of view, spatiality is an uncommon property that often depends on classical reasoning principles. Lemma 2 is geometrically justified, because the Yoneda embedding $\mathscr{Y}$ already provides enough points of the form $\mathscr{Y}(C)$.

If the geometric theory $\mathbb{T}$ in Lemma 2 is classified by the topos $\mathscr{E}$, then the lemma tells us that geometric morphisms from $[\mathscr{C}$, Set $]$ to $\mathscr{E}$ are equivalent to $\mathscr{C}$-indexed diagrams of points of $\mathscr{E}$. In fact, this can be used to show that $[\mathscr{C}$, Set $]$ is exponentiable as a topos, with $\mathscr{E}[\mathscr{C}$, Set $]$ classifying the theory of $\mathscr{C}$-indexed diagrams of points of $\mathscr{E}$ (see e.g. [28]).

We should also ask what are the geometric morphisms to $[\mathscr{C}$, Set $]$, i.e., its points.

Definition 3. Let $\mathscr{C}$ be a small category, and $F: \mathscr{C}^{o p} \rightarrow$ Set a presheaf. $F$ is flat if it has the following properties

1. For some $C \in \mathscr{C}_{0}, F(C)$ is inhabited.

2. If $x_{i} \in F\left(C_{i}\right)(i=1,2)$, then there are morhisms $f_{i}: C_{i} \rightarrow D$ in $\mathscr{C}$, and $y \in F(D)$, such that $x_{i}=$ $F\left(f_{i}\right)(y)$.

3. If $f, g: C \rightarrow D$ in $\mathscr{C}$ and $y \in F(D)$ with $F(f)(y)=F(g)(y)$, there is is some morphism $h: D \rightarrow E$ such that $h f=h g$, and some $z \in F(E)$ such that $y=F(h)(z)$.

The theory of flat presheaves over $\mathscr{C}$ is geometric, and its models are equivalent to the points of $[\mathscr{C}$, Set $]$ (see e.g. [45]).

The role of the flatness conditions becomes clearer if one considers the Grothendieck construction, which turns a presheaf $F$ into a category $\int F$, the so-called category of elements of $F$. Its objects are pairs $(C, u)$ with $u \in F(C)$, and a morphism from $(C, u)$ to $(D, v)$ is an arrow $f: C \rightarrow D$ such that $u=F(f)(v)$. Then $F$ is flat iff $\int F$ is filtered, and in that case $F$ can be thought of as a filtered diagram of representable presheaves $\mathscr{Y}(C)$ (which are themselves flat). In fact, as point of $[\mathscr{C}$, Set $], F$ is a filtered colimit of representables.

We shall mostly use this in the case where $\mathscr{C}$ is a poset $P$.

Lemma 4. For a poset $P$, flat presheaves $F: P^{o p} \rightarrow$ Set correspond up to isomorphism with ideals of $P$.

Proof. Let $F$ be a flat presheaf, and $p \in P$. By Definition 3(2) $F(p)$ contains at most one element. By this observation, we identify the set of objects of $\int F$ with a subset $I \subseteq P$, where $p \in I$ iff there exists an (automatically unique) element $x \in F(p)$, i.e., $(p, x) \in \int F$. Under the identification of $\int F$ with $I$, the arrows of $\int F$ translate to the order relation of $P$, restricted to $I$. By conditions (1) and (2) of Definition 3 . $I$ is non-empty and upward directed. Condition (3) automatically holds for posets. The set $I$ is downward closed as $F$ is a presheaf. For a flat functor $F$, we have identified the category $\int F$ with an ideal $I$ of $P$. Conversely, to each ideal $I$ of $P$, we can associate a flat functor $F$, which has $I$ as its category of elements.

Then the flat presheaves are simply the ideals of $P$, and we see that the functor topos $[P$, Set $]$ is the topos of sheaves over the locale Idl $P$ whose points are the ideals of $P$ and whose opens are the Scott opens of the ideal completion, or, equivalently, the Alexandrov opens (up-closed subsets) of $P$. 
A technique that we shall find useful in various places is a simple form of iterated forcing. Suppose $\mathscr{D}$ is an internal category in $[\mathscr{C}$, Set $]$, hence a functor from $\mathscr{C}$ to Cat. A topos $[\mathscr{D},[\mathscr{C}$, Set $]]$ of internal diagrams can be defined, together with a geometric morphism to $[\mathscr{C}$, Set $]$. A point of it is a pair $(F, G)$ where $F$ is flat presheaf over $\mathscr{C}$ and $G$ is a flat presheaf over $\mathscr{D}(F)$, i.e. $\operatorname{colim}_{(C, x)} \mathscr{C}(C)$, the filtered colimit of the diagram corresponding to $F$.

It turns out (see e.g. [30, C2.5]) that $[\mathscr{D},[\mathscr{C}$, Set $]]$ too is a presheaf topos, over the category $\mathscr{C} \ltimes \mathscr{D}$ defined as follows. Its objects are pairs $(C, D)$ with $C$ an object of $\mathscr{C}$ and $D \in \mathscr{D}_{0}(C)$, where $\mathscr{D}_{0}: \mathscr{C} \rightarrow$ Set is the object of objects of $\mathscr{D}$. An arrow $(f, g):(C, D) \rightarrow\left(C^{\prime}, D^{\prime}\right)$ in $\mathscr{C} \ltimes \mathscr{D}$ is given by an arrow $f: C \rightarrow C^{\prime}$ in $\mathscr{C}$ and an arrow $g \in \mathscr{D}_{1}\left(C^{\prime}\right), g: \mathscr{D}_{0}(f)(D) \rightarrow D^{\prime}$.

As an example, consider the case where $\mathscr{D}$ is discrete, corresponding to an object $U: \mathscr{C} \rightarrow$ Set in $[\mathscr{C}$, Set $]$. Then the geometric morphism $[\mathscr{D},[\mathscr{C}$, Set $]] \rightarrow[\mathscr{C}$, Set $]$ is the local homeomorphism corresponding to $U$. We thus see that the external view of the internal discrete locale $U$ is also given by a presheaf topos, on $C \ltimes U$. Its objects are pairs $(C, u)(C$ an object of $\mathscr{C}, u \in U(C))$, and a morphism from $(C, u)$ to $(D, v)$ is a morphsm $f: C \rightarrow D$ such that $v=U(f)(u)$.

\subsection{A result on exponentiability}

This subsection presents a general result (Theorem 7) on exponentiability. It is not specifically about geometricity, but is related because geometricity would like to replace the non-geometric construction of the frame $\mathscr{O} X$ by the locale exponential $\mathbb{S}^{X}$, which has the same points $\left(\mathscr{O} X\right.$ is the set of points of $\left.\mathbb{S}^{X}\right)$ and is geometric. However, the exponential exists only if $X$ is locally compact, and so we are going to be interested in local compactness in order to define the elements of the frame geometrically.

In fact, the following known theorem [24] holds for locales in any elementary topos.

Theorem 5. Let $X$ be a locale, then the following are equivalent:

1. $X$ is locally compact.

2. The functor $(-) \times X:$ Loc $\rightarrow$ Loc has a right adjoint $(-)^{X}$.

3. The exponential $\mathbb{S}^{X}$ exists, where $\mathbb{S}$ denotes the Sierpiński locale.

We are going to build up to Theorem 6, stating that 'locally perfect maps compose', where $p: Y \rightarrow X$ is locally perfect if the corresponding internal locale $\underline{Y}$ in $\operatorname{Sh}(X)$ is locally compact ${ }^{6}$ The precise form of our result is that if $X$ is a locally compact locale, and $p: Y \rightarrow X$ is locally perfect, then $Y$ is locally compact. However, this can be relativized. If also $q: Z \rightarrow Y$ is locally perfect, then by working in $\operatorname{Sh}(X)$ we find that $p q$ is locally perfect.

We shall apply this to the spectrum of an internal $\mathrm{C}^{*}$-algebra to show that its external description is a locally compact locale.

Consider $p: Y \rightarrow X$ as above, and assume for a moment that the exponential $\mathbb{S}^{Y}$ exists. A point of $\mathbb{S}^{Y}$ is equivalent to a map $Y \rightarrow \mathbb{S}$, which corresponds to an open $U \in \mathscr{O} Y$. By assumption $\underline{Y}$ is locally compact in $\operatorname{Sh}(X)$, so the exponential $\underline{\mathbb{S}} \underline{\underline{Y}}$ exists in $\operatorname{Sh}(X)$, where $\underline{\mathbb{S}}$ denotes the internal Sierpiński locale. Using the fact that $\mathbf{L o c}_{\mathrm{Sh}(X)}$ is equivalent to $\mathbf{L o c} / X$, the locale $\underline{\mathbb{S}} \underline{\underline{Y}}$ has an external description by a locale map $q: \mathbb{S}_{X}^{Y} \rightarrow X$, for some locale $\mathbb{S}_{X}^{Y}$. The external description of $\underline{\mathbb{S}}$ is the projection $\pi_{2}: \mathbb{S} \times X \rightarrow X$. As

\footnotetext{
6 The terminology is debatable, and will depend on whether one calls p perfect or proper for the situation where $\underline{Y}$ is compact. In the present paper we are following Johnstone's usage [27, where a map $f: X \rightarrow Y$ between topological spaces is called locally perfect if the following condition holds. If $U \in \mathscr{O} X$ and $x \in U$, then there exists an open neighborhood $V$ of $x$, an open neighborhood $N$ of $f(x)$, and a set $K \subseteq X$, such that $K \subseteq U \cap f^{-1}(N)$ and for each $b \in N$, the fibre $K_{b}$ is compact. He shows that if $f$ is locally perfect, then $f_{*}(X)$ is a locally compact locale in $\operatorname{Sh}(Y)$, and the converse holds if $Y$ satisfies the $T_{D}$ separation property.
} 
exponentiation of locales is geometric [41, Sec 10], the fibre $q^{*}(\{x\})$ over a point $x$ in $X$ is given by $\mathbb{S}^{Y_{x}}$, where we write $Y_{x}$ for the fibre $x^{*} Y=p^{-1}(\{x\})$.

An open $U \in \mathscr{O} Y$ and a point $x \in X$ give an open $U_{x}$ in the fibre $Y_{x}$, as in the figure below. This in turn is equivalent to a map $Y_{x} \rightarrow \mathbb{S}$, which is a point of $q^{*}(\{x\})$. This suggests that the global points of $\mathbb{S}^{Y}$ correspond exactly to the global sections of the bundle $q: \mathbb{S}_{X}^{Y} \rightarrow X$. That is, global points of $\mathbb{S}^{Y}$ correspond to maps $\sigma: X \rightarrow \mathbb{S}_{X}^{Y}$ such that $q \circ \sigma=\mathrm{id}_{X}$.

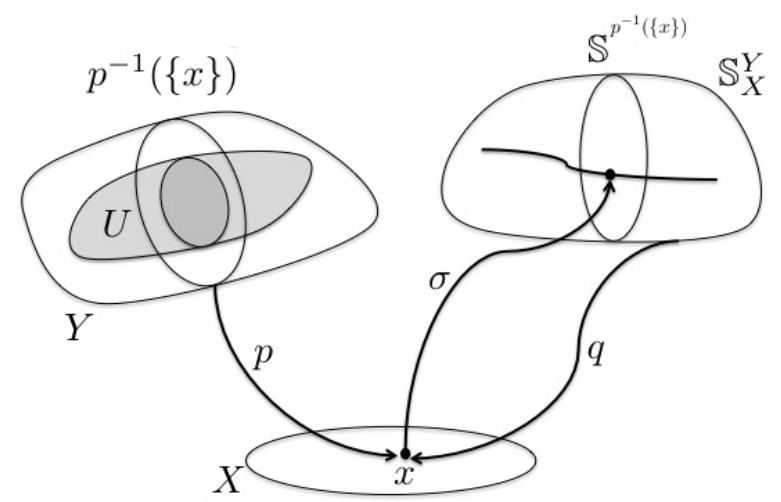

The informal reasoning above can be made precise in order to prove that for a locally compact $X$, and $\underline{Y}$ a locally compact in $\operatorname{Sh}(X)$, with external description $p: Y \rightarrow X$, the locale $Y$ is locally compact. We define locales by providing their geometric theories (of generalized points) and we define continuous maps as constructively described transformations of points of one locale to points of another locale; see [45, Sec. 4.5].

Theorem 6 (locally perfect maps compose). If $X$ is a locally compact locale, and $\underline{Y}$ a locally compact locale in $\operatorname{Sh}(X)$, with external description $p: Y \rightarrow X$, then $Y$ is locally compact.

Proof. The locale $Y$ is the locale with (generalized) points the pairs $(x, t)$ such that $x \in X, t \in Y_{x}$. That is, we view the map $p$ as a bundle; see [48] and the discussion of the bundle picture in Subsection 2.2 As before, $\mathbb{S}_{X}^{Y}$ denotes the external description of the internal locale $\mathbb{S}^{\underline{Y}}$ in $\operatorname{Sh}(X)$. Since the exponential is geometric [41, Sec. 10.3], the generalized points of $\mathbb{S}_{X}^{Y}$ are the pairs $(x, w)$ such that $x \in X, w \in \mathbb{S}^{Y_{x}}$. The internal evaluation map ev $: \underline{\mathbb{S}} \underline{Y} \times \underline{Y} \rightarrow \underline{\mathbb{S}}$, part of the geometric structure of $\underline{\mathbb{S}} \underline{\underline{Y}}$, must then correspond to a map ev : $\mathbb{S}_{X}^{Y} \times_{X} Y \rightarrow \mathbb{S}$ given by $((x, w), y) \mapsto w(y)$. We define $E$ as the locale with (generalized) points those $\sigma: X \rightarrow \mathbb{S}_{X}^{Y}$ such that $q \circ \sigma=\mathrm{id}_{X}$. Here local compactness of $X$ allows us to define the exponential $\left(\mathbb{S}_{X}^{Y}\right)^{X}$, and an equalizer captures the section condition to give a sublocale. To define the map ev $: E \times Y \rightarrow \mathbb{S}$, we first define the map $E \times Y \rightarrow \mathbb{S}_{X}^{Y} \times{ }_{X} Y$ by $(\sigma, y) \mapsto(\sigma(p y), y)$ and then compose it with the map ev $: \mathbb{S}_{X}^{Y} \times_{X} Y \rightarrow \mathbb{S}$.

To complete the proof, we require that if $g: Z \times Y \rightarrow \mathbb{S}$, then there is a unique $\tilde{g}: Z \rightarrow E$ such that $g=\operatorname{ev} \circ\left(\tilde{g} \times \mathrm{id}_{Y}\right)$. This condition amounts to saying that for all $z, y$,

$$
g(z, y)=w(y), \text { where } \tilde{g}(z)(p y)=(p y, w) .
$$

Assuming this condition, consider $\tilde{g}(z)(x)=(x, w)$, for some $w$ in $\mathbb{S}^{Y_{x}}$. For all $y$ in $Y_{x}$ we must have $\tilde{g}(z)(p y)=\tilde{g}(z)(x)=(p y, w)$, and by the condition $w(y)=g(z, y)$. Hence $w$ is uniquely determined for each $x$, and so $\tilde{g}$ is unique. Reversing the argument, and relying heavily of geometricity, we see that it leads to a definition of $\tilde{g}$, given $g$. 
Theorem 6 holds in a much more abstract categorical form. This can be applied when the base $X$ is a non-localic topos that is exponentiable in the category of toposes - our prime example will be a topos of the form $[\mathscr{C}$, Set $]$ where $\mathscr{C}$ is not a poset.

Theorem 7. Let $\mathscr{C}$ be a category with finite limits, and let $X$ be an exponentiable object in $\mathscr{C}$. Let $p: Y \rightarrow X$ be an object $\underline{Y}$ of $\mathscr{C} / X$, let $Z$ be an object of $\mathscr{C}$, and suppose the exponential $\underline{Z}^{\underline{Y}}$ exists in $\mathscr{C} / X$. We shall write it as $q: \bar{Z}_{X}^{Y} \rightarrow X$. Then $Z^{Y}$ exists in $\mathscr{C}$.

Proof. By the considerations above Theorem 6, we arrive at the following candidate for the exponential $Z^{Y}$. Take the equalizer

$$
E \stackrel{e q}{\longrightarrow}\left(Z_{X}^{Y}\right)^{X} \underset{\ulcorner X\urcorner \circ !}{\stackrel{q^{X}}{\longrightarrow}} X^{X},
$$

where $\ulcorner X\urcorner: 1 \rightarrow X^{X}$ denotes the transpose of the identity arrow of $X$. Note that the exponentials $\left(Z_{X}^{Y}\right)^{X}$ and $X^{X}$ exist in $\mathscr{C}$ by exponentiability of $X$. Also note that the global points of $E$ are exactly the global sections of $q$. Next, we need to find a suitable evaluation map ev $: E \times Y \rightarrow Z$. For the definition of $e v$ we will make use of the internal evaluation arrow $\underline{Z}^{\underline{Y}} \times \underline{Y} \rightarrow \underline{Z}$. Externally this gives the following commuting triangle:

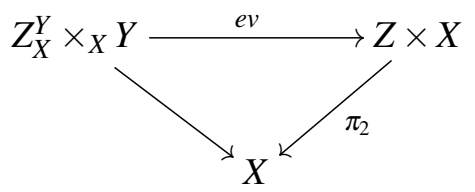

With some abuse of notation, we denote the map $\pi_{1} \circ \mathrm{ev}: Z_{X}^{Y} \times_{X} Y \rightarrow Z$ again by $\mathrm{ev}$. For the next step in defining the evaluation map, the diagram given below is commutative by the definition of $E$.

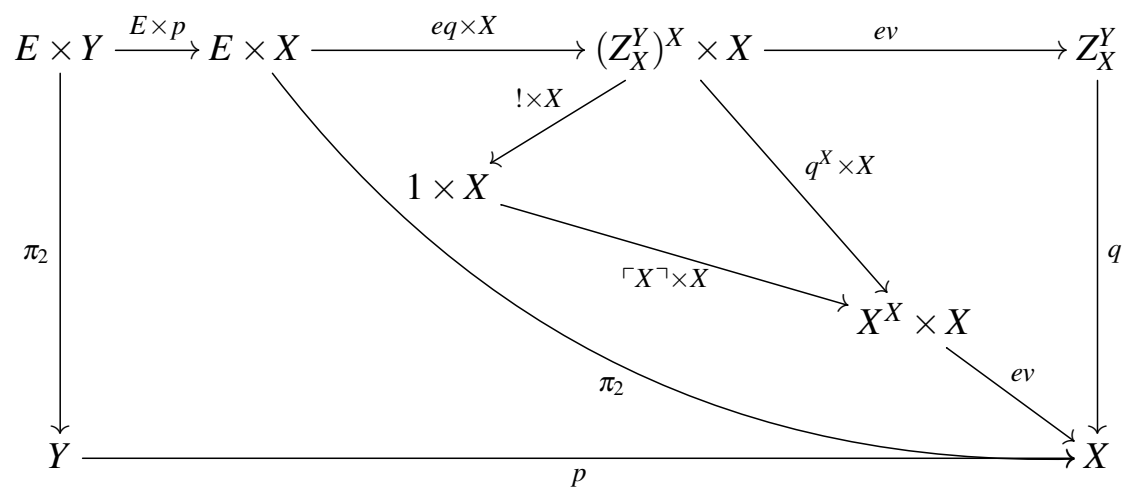

The evaluation maps and exponentials in this diagram exist because of the exponentiability of $X$. By the universal property of pullbacks this diagrams yields an arrow $E \times Y \rightarrow Z_{X}^{Y} \times_{X} Y$. Taking the composition with $e v: Z_{X}^{Y} \times_{X} Y \rightarrow Z$ coming from the internal exponential $\underline{Z}^{\underline{Y}}$ gives the desired evaluation map ev $: E \times Y \rightarrow Z$.

It remains to check that this map satisfies the desired universal property. Maps $Z \times Y \rightarrow Z$ correspond bijectively with maps $(Z \times X) \times{ }_{X} Y \rightarrow Z \times X$ over $X$. By the existence of the internal exponent $\underline{Z}^{\underline{Y}}$ the latter maps correspond bijectively with maps $Z \times X \rightarrow Z_{X}^{Y}$ over $X$. Using exponentiability of $X$ maps $Z \times X \rightarrow Z_{X}^{Y}$ correspond bijectively with maps $Z \rightarrow\left(Z_{X}^{Y}\right)^{X}$. The maps $Z \times X \rightarrow Z_{X}^{Y}$ that are maps over $X$ precisely correspond to the maps $Z \rightarrow\left(Z_{X}^{Y}\right)^{X}$ that factor through $E$. This proves that $E$ is indeed an exponential $Z^{Y}$. 
In particular it follows from the theorem that if $\underline{Y}$ is exponentiable in $\mathscr{C} / X$, then $Y$ is exponentiable in $\mathscr{C}$. Theorem 6 is the special case where $\mathscr{C}$ is the category of locales.

\section{Toposes and Quantum Theory}

The topos approaches to quantum theory were inspired by the work of Butterfield and Isham [25, 20, 6, 7]. The original "BI" approach, or presheaf approach (as it is formulated using a topos of presheaves) was subsequently developed by Döring and Isham [15]. In the present paper we are particularly interested in the different formulation of [21], which we call the copresheaf approach. What these two approaches share is their use, for a given quantum system described by a $\mathrm{C}^{*}$-algebra, of a topos whose internal logic embodies the idea of fixing a generic context, or classical perspective on that system. Thus in the internal logic, the system has a classical phase space. Our aim here is to show how this internal phase space can be represented externally by a bundle, whose fibres are the phase spaces for individual contexts.

In the approaches cited, a unital $\mathrm{C}^{*}$-algebre ${ }^{7} A$ is studied through the poset $\mathscr{C}(A)$ of commutative unital $C^{*}$-subalgebras of $A$, ordered by inclusion, and using a topos of functors from $\mathscr{C}(A)$ to Set.

At this point the presheaf and copresheaf approaches diverge, using contravariant and covariant functors respectively. We shall focus now on the copresheaf approach with its topos $[\mathscr{C}(A)$, Set]. To study the operator algebra $A$ from the perspectives of its commutative $\mathrm{C}^{*}$-subalgebras, it is replaced by the covariant functor $\underline{A}: \mathscr{C}(A) \rightarrow$ Set, $\underline{A}(C)=C$, where the arrows are mapped to inclusions. In the topos $[\mathscr{C}(A)$, Set $], \underline{A}$ becomes an internal unital commutative $\mathrm{C}^{*}$-algebra.

From the internal perspective of the topos, the quantum-mechanical observables, now in the form of the commutative $\mathrm{C}^{*}$-algebra $\underline{A}$, look more like the observables of a classical physical theory. Crucial for this is the topos-valid version of Gelfand duality, described in the work of Banaschewski and Mulvey [3, 2,4] as a duality between the category of unital commutative $C^{*}$-algebras and the category of compact, completely regular locales. A more explicit and fully constructive description of this Gelfand duality is given in [9, 10]. By this duality, $\underline{A}$ is isomorphic to the $C^{*}$-algebra of continuous, complex-valued functions on a certain compact, completely regular locale $\underline{\Sigma}$, the spectrum of $\underline{A}$, which we think of as a phase space. For further discussion of the ideas of the topos approach, such as the treatment of states, see $[21,23,22,8,16]$. We also mention exciting recent work which reconstructs the Jordan algebra structure from the spectral object [18, 19, 14, 13, 12].

In this section we apply the geometricity ideas of the previous section to the topos approach described in [21]. In particular, we concentrate on the description of the spectrum of internal commutative unital $\mathrm{C}^{*}$-algebras in toposes. The first subsection examines the geometricity of parts of the definition of $\mathrm{C}^{*}$ algebra. This is a central issue, since the $\mathrm{C}^{*}$-algebra structure itself is not geometric, so it must be replaced by a geometric structure that, while not being the whole $\mathrm{C}^{*}$-algebra, nonetheless supports a geometric construction of the spectrum.

The second subsection examines the geometricity of the construction of the spectrum. The third subsection is concerned with finding an explicit description of the spectrum of the algebra $\underline{A}$ in the particular topos $[\mathscr{C}(A)$, Set $]$, which is central to the topos approach. The fourth subsection generalizes this description to spectra of commutative unital $C^{*}$-algebras in functor categories in general. In the fifth subsection we consider extending the copresheaf approach to algebraic quantum field theory. Throughout the emphasis will be on the role of geometric logic.

\footnotetext{
${ }^{7}$ In the presheaf approach the smaller class of von Neumann algebras are typically considered, rather than arbitrary unital $\mathrm{C}^{*}$-algebras.
} 


\section{1 $C^{*}$-algebras in toposes}

In a topos $\mathscr{E}$, a $\mathrm{C}^{*}$-algebra $A$ (always assumed unital here) -

1. is an associative, unital algebra over $\mathbb{C}$ with an involution $*$, which is anti-multiplicative $\left((a b)^{*}=\right.$ $\left.b^{*} a^{*}\right)$ and conjugate linear $\left((z a+b)^{*}=\bar{z} a^{*}+b^{*}\right.$, where $\left.z \in \mathbb{C}\right)$,

2. has a norm $\|-\|$, which is submultiplicative and satisfies the axiom $\left\|a a^{*}\right\|=\|a\|^{2}$, and

3. is complete with respect to the norm.

However, the definition is not geometric, for three reasons.

First, with regard to the algebraic structure (1) above, already $\mathbb{C}$ - as set of complex numbers rather than a locale - is not a geometric construction. For a truly geometric account, a $\mathrm{C}^{*}$-algebra would have to be a locale rather than a set. In [4], a $\mathrm{C}^{*}$-algebra is defined as an algebra over the Gaussian numbers $\mathbb{Q}[i]$, upon which completeness then allows the action of $\mathbb{Q}[i]$ to be extended to $\mathbb{C}$. Apart from this, the algebraic part is straightforward. It consists of arrows $\underline{ \pm}, \underline{:}: \underline{A} \times \underline{A} \rightarrow \underline{A}$ for addition and multiplication, an arrow $\underline{*}: \underline{A} \rightarrow \underline{A}$ for the involution, an arrow $\mathbb{Q}[i] \times \underline{A} \rightarrow \underline{A}$ for scalar multiplication, and constants $0,1: \underline{1} \rightarrow \underline{A}$ for the unit and zero element. These arrows are to render all desired diagrams commutative. Note that we made use of the geometricity of $\mathbb{Q}[i]$. In the language of Subsection 2.1, in a formal geometric theory we could declare a sort $k$, add structure and axioms to force it to be isomorphic to $\mathbb{Q} \times \mathbb{Q}$, and then define the appropriate operations as ring with involution.

Second, the norm is not geometric. We shall develop a geometric theory of "commutative G*algebra", more general than unital commutative $\mathrm{C}^{*}$-algebras, but expressing enough of the structure to define the spectrum. For the norm, we shall ignore the condition $\|a\|=0 \rightarrow a=0$, as it is not geometric 8 . so we have only a semi-norm. As explained in [43], the semi-norm as described in [4] with a binary relation $N \subseteq A \times \mathbb{Q}^{+}$can be understood as a map $\|\cdot\|: A \rightarrow \overleftarrow{[0, \infty]}$ taking its values in the upper reals. Then $(a, q) \in N$ if $\|a\|<q$. The third reason, connected to the first one, is that the completeness of $A$ with respect to the norm is not geometric.

What we seek, therefore, is a geometric notion that generalizes commutative $\mathrm{C}^{*}$-algebras, and on which we can still, and geometrically, calculate the spectrum.

There is a geometric core to the definition of $\mathrm{C}^{*}$-algebra, in the notion of semi-normed pre-C*algebra - that is, a $*$-algebra over $\mathbb{Q}[i]$, but dropping completeness and weakening the norm to a seminorm. However, that runs into problems because at a certain point in constructing the spectrum we need to know the the order on the self-adjoints $A_{s a}$, or, alternatively, its positive cone. In a $\mathrm{C}^{*}$-algebra a selfadjoint is (non-strictly) positive iff it is a square, but without completeness we cannot guarantee the vital property that the sum of squares is still a square - for example, 2 might not be a square.

Our way round this is to use the preordered archimedean rings of [9].

Definition 8. A commutative $\mathbb{Q}$-algebra $R$ is called preordered if it has a positive cone, i.e., a subset $P$ that contains all squares and is closed under addition and multiplication by $\mathbb{Q}^{+}$. The preorder is then given by $a \leq b$ if $b-a \in P$.

The preorder $R$ is archimedean if, in addition, for each $a \in R$ there is some $r \in \mathbb{Q}$ such that $a \leq r$.

A commutative $G^{*}$-algebra is a commutativ $\mathbb{9}^{9} \mathbb{Q}[i]-*$-algebra $A$ for which the self-adjoint part $A_{s a}$ is a preordered archimedean ring.

\footnotetext{
${ }^{8}$ When the norm is expressed as a subobject of $A \times \mathbb{Q}^{+}$, the potential non-geometricity of the axiom is visible in $\|a\|=0$ as a universal quantifier over $\mathbb{Q}^{+}$.

${ }^{9}$ In this ad hoc naming, we write ' $G$ ' for 'geometric'. We have not attempted to define non-commutative $\mathrm{G}^{*}$-algebras, since it is not so easy to order the self-adjoints when they don't commute.
} 
Since the definition is geometric, there is a canonical notion of homomorphism between two commutative $\mathrm{G}^{*}$-algebras: a function that preserves the *-algebra structure and positivity.

\subsection{The Gelfand spectrum and normal lattices}

If $A$ is a commutative $C^{*}$-algebra in a topos, then its spectrum $\Sigma_{A}$ is the space of the continuous $*$-algebra homomorphisms $x: A \rightarrow \mathbb{C}$. The classical theory then says that $A$ is isomorphic to the complex algebra of continuous maps from $\Sigma_{A}$ to $\mathbb{C}$, and its self-adjoint part $A_{s a}$ is isomorphic to the real algebra of continuous maps from $\Sigma_{A}$ to $\mathbb{R}$. The topology on $\Sigma_{A}$ is the weak-* topology, and another way to say this is that a subbasis of opens is provided by the sets of the form $\{x \mid x(a)>0\}$ for $a \in A$ self-adjoint.

This suggests that if we want a geometric description of the points of the spectrum, we should use the elements $a \in A_{s a}$ to form propositional symbols (let us say $D(a)$ ), and add axioms to say that in this system $D(a)$ behaves like $\{x \mid x(a)>0\}$. This was done in [4].

Coquand [9] defines, for any preordered archimedean ring, the spectrum (which he calls the maximal spectrum) in a point-free way.

If $A$ is a commutative $C^{*}$-algebra, $A$ is a commutative $\mathrm{G}^{*}$-algebra by restricting $\mathbb{C}$ to $\mathbb{Q}[i]$ and defining its positive cone to be the set of all squares. This is closed under addition and multiplication by positive rationals. Also, the archimedean property follows from the existence of the norm. Then [10] its point-free Gelfand spectrum is isomorphic to the spectrum constructed in [9].

As mentioned before, a point-free approach will give axioms characterizing the behaviour of formal symbols $D(a)\left(a \in A_{s a}\right)$ with intended meaning $\{x \mid x(a)>0\}$. The axioms in [9] are:

$$
\begin{aligned}
D(a) \wedge D(-a) & \vdash \\
D(a) & \vdash \\
D(a+b) & \vdash D(a) \vee D(b) \\
\top & \text { if } a \leq 0(1) \\
D(a b) & \vdash(D(a) \wedge D(b)) \vee(D(-a) \wedge D(-b)) \\
D(a) & \vdash \bigvee_{0<r \in \mathbb{Q}} D(a-r) .
\end{aligned}
$$

These are all intuitively clear in terms of the intended meaning. For example, (1a) says that for no $x$ can we have both $x(a)>0$ and $x(-a)=-x(a)>0$, while (2) says that if $x(a)>0$, then $x(a)>r$ for some $r>0$.

The fact that these axioms are enough is not at all obvious, and takes up some substantial, non-trivial calculations [9]. The upshot is that each point $x$ of the spectrum can be described geometrically by the set of those elements $a \in A_{s a}$ for which $x(a)>0$. In fact, we could take it (but we shall modify this view) that that set $i$ s the point $x$. Such a set must conform with the axioms. For example, it cannot contain both $a$ and $-a$.

We do not wish to recap those substantial calculations, but there is an important structural part of the development of which we shall make considerable use. The six axioms above (12) are a propositional geometric theory, with propositional symbols $D(a)$ indexed by elements $a$ of $A_{s a}$. As mentioned in Section 2.1, it can be used to present a frame $F_{A}$, with the $D(a)$ as generators and the axioms as relations. Then the points of the spectrum correspond to completely prime filters of the frame. However, the first group of axioms (1) do not use infinitary disjunctions, and so could be taken as presenting a finitary distributive lattice. 
Definition 9. Let $A$ be a commutative $G^{*}$-algebra. We write $L_{A}$ for the distributive lattice presented by generators $D(a)\left(a \in A_{s a}\right)$ subject to the above relations (1). (In [9], $L_{A}$ is referred to as $\operatorname{Spec}_{r}\left(A_{s a}\right)$.)

Unlike the frame $F_{A}$, the lattice $L_{A}$ is constructed geometrically from $A$. This is not noted explicitly in [9], but is a consequence of the way it is presented by generators and relations because geometric constructions include free algebras, and generating and factoring out congruences. The techniques are as described in [36]. It is, in fact, possible to give a description that is more concrete than that of the general universal algebra, though we shall not need it here. [42] shows how to construct, geometrically, a free distributive lattice as a quotient of a double finite powerset, in this case $\mathscr{F} \mathscr{F} A_{s a}$. [9] then gives an explicit concrete description of when, in $L_{A}$, we have

$$
D\left(a_{1}\right) \wedge \cdots \wedge\left(a_{n}\right) \leq D\left(b_{1}\right) \vee \cdots \vee D\left(b_{m}\right) .
$$

It follows that points $x$ of the spectrum can also be represented as subsets of $x \subseteq L_{A}$. Each subset corresponds to a map from $L_{A}$ to the subobject classifier $\Omega$, and respecting the axioms $(1)$ then amounts to saying that this map preserves meets and joins (all finitary), in other words that $x$ is a prime filter of $L_{A}$. It is a filter if it is up-closed and closed under finite meets, and it is prime if whenever it contains a finite join, then it also contains one of the elements joined.

At this point we can thus describe the points of the spectrum of $A$ geometrically as prime filters $x$ of $L_{A}$, not arbitrary ones, but those respecting axiom (2), in other words such that if $D(a) \in x$ then $D(a-r) \in x$ for some $0<r \in \mathbb{Q}$. This is slightly awkward because, although we have reduced much to the lattice, we still have to refer explicitly to the elements of $A$. The next stage will remove this awkwardness, and at the same time give access to a general geometric treatment of compact regular locales.

Definition 10. A distributive lattice $L$ is said to be normal if whenever $a \vee b=\top$ then there are $x$ and $y$ such that $a \vee y=x \vee b=\top$ and $x \wedge y=\perp$. Defining $a^{\prime} ₹ a\left(a^{\prime}\right.$ well inside a) if there is $y$ such that $a \vee y=\top$ and $a^{\prime} \wedge y=\perp$, then another way to express normality is that if $a \vee b=\top$, then there is some $a^{\prime} \gtrless a$ with $a^{\prime} \vee b=\top$. We also write $\downarrow$ a for the set $\left\{a^{\prime} \mid a^{\prime} \gtrless a\right\}$.

If $L$ is a normal distributive lattice, then a prime filter $x \subseteq L$ is regular if whenever $a \in x$, then $a^{\prime} \in x$ for some $a^{\prime}<a$.

The theory of regular ideals of a normal distributive lattice $L$ is geometric. We could describe this by a propositional theory along the lines of that used earlier for $\Sigma_{A}$, but to illustrate the use of geometric mathematics we present a predicate theory that describes the regular ideals directly. It has a predicate symbol $x \subseteq L$, and axioms as follows.

$$
\begin{gathered}
\top \vdash x(\top) \\
x(a) \wedge x(b) \vdash_{a b} x(a \wedge b) \\
x(\perp) \vdash \perp \\
x(a \vee b) \vdash_{a b} x(a) \vee x(b) \\
x(a) \vdash_{a}\left(\exists a^{\prime}\right)\left(a^{\prime} \gtrless a \wedge x\left(a^{\prime}\right)\right)
\end{gathered}
$$

(Note that the logical symbols $\top, \wedge, \perp, \vee$ are overloaded here, denoting both operators in the lattice $L$ and logical connectives. The two usages are syntactically quite different, though clearly the axioms set up a semantic connection between them.) Axioms $3 \mathrm{a} 3 \mathrm{~b}$ say that $x$ is a filter, $3 \mathrm{c} 3 \mathrm{~d}$ ) that it is prime, and $3 \mathrm{e}$ ) that it is regular.

This is presented as a predicate theory, and so, in principle, has a classifying topos that might not be localic. However, it is localic, and this is evident from the fact that no new sorts are declared in the 
signature. The cautious reader can write down an equivalent propositional theory explicitly - this process is discussed in [45] for the Dedekind reals.

We write RSpec $L$ (the regular spectrum of $L$ ) for the locale just defined, whose points are the regular prime filters of $L$.

Coquand (9] proved (i) $L_{A}$ is normal, and (ii) axiom (2) is equivalent to the regularity axiom $a \vdash$ $\bigvee_{a^{\prime} \approx a} a^{\prime}$. This is important as it implies that the spectrum $\Sigma_{A}$ is isomorphic to $\operatorname{RSpec} L_{A}$, the regular spectrum of $L_{A}$.

This completes our geometric construction of the spectrum from the $\mathrm{C}^{*}$-algebra. We replaced the non-geometric commutative $\mathrm{C}^{*}$-algebra by the more general and geometric commutative $\mathrm{G}^{*}$-algebra. The spectrum of this $\mathrm{G}^{*}$-algebra $A$ is constructed geometrically by first constructing the normal distributive lattice $L_{A}$ and subsequently constructing its regular spectrum.

If $f: A \rightarrow B$ is a homomorphism of commutative $\mathrm{G}^{*}$-algebras, then geometricity ensures that it gives a lattice homomorphism $L_{f}: L_{A} \rightarrow L_{B}$, and (contravariantly) a map $\Sigma_{f}: \Sigma_{B} \rightarrow \Sigma_{A}$. If $y$ is a regular prime filter of $L_{B}$, then $\Sigma_{f}(y)$ is its inverse image $L_{f}^{-1}(y)$. If $f$ is an inclusion, we shall generally write $L_{A B}$ for $L_{f}$ and $\rho_{B A}$ for $\Sigma_{f}$.

In the next two lemmas we shall consider formal expressions $\phi\left(x_{1}, \ldots, x_{n}\right)=\phi\left(x_{i}\right)_{1}^{n}$ built using finite meets and finite joins from generators $D\left(x_{i}\right)$, where $x_{i}$ is a variable ranging over $A_{s a}$. From [9] we know that $\phi\left(a_{i}-r\right)_{1}^{n}<\phi\left(a_{i}\right)_{1}^{n}$ if $0<r \in \mathbb{Q}$. [9, Corollary 1.7] also shows that if $\phi$ is a finite join of generators, and $\phi\left(a_{i}\right)_{1}^{n}=1$ in $L_{A}$ for some elements $a_{i} \in A_{s a}$, then $\phi\left(a_{i}-r\right)_{1}^{n}=1$ for some $0<r \in \mathbb{Q}$. This clearly extends to the case where $\phi$ is a finite meet of finite joins of generators, and hence to arbitrary distributive lattice expressions.

Lemma 11. Let $A$ be a commutative $G^{*}$-algebra, and suppose $v \gtrless u=\phi\left(a_{i}\right)_{1}^{n}$ in $L_{A}$. Then there is some $0<r \in \mathbb{Q}$ such that $v \leq \phi\left(a_{i}-r\right)_{1}^{n}$.

Proof. We have $u \vee w=1$ and $v \wedge w=0$ for some $w$, and - extending the list of $a_{i}$ s as necessary - we can write $w=\psi\left(a_{i}\right)_{1}^{n}$. By the previous discussion we can find $0<r \in \mathbb{Q}$ such that $\phi\left(a_{i}-r\right)_{1}^{n} \vee \psi\left(a_{i}-r\right)_{1}^{n}=1$, and then, knowing that $\psi\left(a_{i}-r\right)_{1}^{n} \leq w$, we conclude that $v \leq \phi\left(a_{i}-r\right)_{1}^{n}$.

The following lemma will be important when we come to describe the external spectrum.

Lemma 12. Let $f: A \rightarrow B$ be a homomorphism of $G^{*}$-algebras, and suppose $u \in L_{A}$ and $v<L_{f}(u)$ in $L_{B}$. Then there is some $u^{\prime} \gtrless u$ such that $v \leq L_{f}\left(u^{\prime}\right)$.

Proof. Writing $u=\phi\left(a_{i}\right)_{1}^{n}$, we find that $v<\phi\left(f\left(a_{i}\right)\right)_{1}^{n}$. Applying Lemma 11 , we can find $0<r \in \mathbb{Q}$ such that $v \leq \phi\left(f\left(a_{i}\right)-r\right)_{1}^{n}=L_{f}\left(u^{\prime}\right)$ where $u^{\prime}=\phi\left(a_{i}-r\right)_{1}^{n} ₹ u$.

The opens of RSpec $L$ are described in [21] as the regular ideals of $L$, i.e. those ideals $I$ such that if $\downarrow a \subseteq I$ then $a \in I$. This follows from the coverage theorem, a general result of topos-valid locale theory, but unfortunately it is not geometric. The problem lies in the regularity condition, which amounts to

$$
\left(\forall a^{\prime}\right)\left(a^{\prime}<a \rightarrow I\left(a^{\prime}\right)\right) \vdash_{a} I(a)
$$

where the left-hand side is not a geometric formula.

In order to gain access to geometric methods, we replace the regular ideals by the "rounded $₹-$ ideals". They differ in the way they use elements of $L$ to represent opens: a regular ideal comprises those elements of $L$ that are less than the open, whereas the $₹$-ideal comprises those that are well inside. 
The concept of rounded ₹-ideal is geometric, and so there is a locale $\operatorname{RIdl}(L, \gtrless)$ (or, for short, $\operatorname{RIdl} L)^{10}$ whose points are the rounded $₹$-ideals. This much follows [40] from the simple fact that $(L, \gtrless)$ is a continuous information system — that is, $₹$ is an idempotent relation: transitive $(\gtrless \circ \gtrless \subseteq \gtrless)$ and interpolative $(<0<\supseteq<)$.

What is important in our situation is that the points of the locale RIdl $L$ form a frame, and indeed the frame of opens for RSpec $L$. This is expressed succinctly in Theorem 15, that RIdlL is the locale exponential $\mathbb{S}^{\mathrm{RSpec} L}$, which allows us to use Theorem 5 .

Given any idempotent relation $<$ on a set $X$, [40] in effect defines a rounded $<$-ideal to be a model of the geometric theory with one predicate symbol $I \subseteq X$ and axioms

$$
\begin{aligned}
& a^{\prime}<a \wedge I(a) \vdash_{a^{\prime} a} I\left(a^{\prime}\right) \\
& \top \vdash(\exists a) I(a) \\
& I\left(a^{\prime}\right) \wedge I\left(a^{\prime \prime}\right) \vdash_{a^{\prime} a^{\prime \prime}}(\exists a)\left(a^{\prime}<a \wedge a^{\prime \prime}<a \wedge I(a)\right)
\end{aligned}
$$

The corresponding locale is $\operatorname{RIdl}(X,<){ }^{11}$ and its opens are the rounded upsets of $\mathrm{X}$ under $<$.

The proof of the following theorem is instructive. Although the theorem is stated entirely for locales, the proof is fairly simple if one digresses into non-localic toposes. Note that the discussion in Section 2.2 of opfibrations holds equally for maps (geometric morphisms) between toposes. A map from $\mathbb{S}$ to a topos $\mathscr{E}$ is a pair of points of $\mathscr{E}$, together with a specialization morphism between them.

Theorem 13. Let $X$ be a locale, and $(P,<)$ a continuous information system in $\operatorname{Sh}(X)$. Let $p: Y \rightarrow X$ be the external description of the internal locale $\operatorname{RIdl}(P,<)$. Then $p$ is an opfibration.

Proof. Let $\mathscr{E}$ be the classifying topos for the theory of continuous information systems $(Q,<)$, and let $q: \mathscr{F} \rightarrow \mathscr{E}$ be the bundle in which the fibre over $(Q,<)$ is the rounded ideal completion of $(Q,<)$. Hence $\mathscr{F}$ classifies triples $(Q,<, J)$ where $(Q,<)$ is a continuous information system and $J$ is a rounded ideal for it. We first show that $q$ is an opfibration.

Let $f:\left(Q_{\perp},<\right) \rightarrow\left(Q_{\top},<\right)$ be a homomorphism of continuous information systems, in other words, a function $f: Q_{\perp} \rightarrow Q_{\top}$ that preserves $<$. By [46, Proposition 2.10], we obtain a map $\operatorname{RIdl}(f)$ : $\operatorname{RIdl}\left(Q_{\perp},<\right) \rightarrow \operatorname{RIdl}\left(Q_{\top},<\right)$, mapping $J \mapsto \downarrow f(J)$. We have $\operatorname{RIdl}(f)(J) \sqsubseteq J^{\prime}$ iff $f$ extends to a homomorphism (necessarily unique) from $\left(Q_{\perp},<, J\right)$ to $\left(Q_{\top},<, J^{\prime}\right)$, and from this it follows that the map $\left(\left(Q_{\perp},<\right),\left(Q_{\top},<\right), J\right) \mapsto\left(Q_{\perp},<, J, Q_{\top},<, \operatorname{RIdl}(f)(J)\right)$ provides the left adjoint required for an opfibration; see Proposition 1 .

Returning to the situation as in the statement, by the definition of classifying topos the continuous information system in $\operatorname{Sh}(X)$ gives a map $(P,<): X \rightarrow \mathscr{E}$, and $p$ is the pullback of $q$ along it. From the geometricity of the definition of opfibration, we see that the property is preserved under pullback.

To see that $₹$ is an idempotent relation (which is well known), first note that in any distributive lattice $L$ we have that if $b^{\prime} \leq a^{\prime}<a \leq b$ then $b^{\prime}<b$, and if $a^{\prime}<a$ then $a^{\prime} \leq a$. From this is follows that $₹$ is transitive. If moreover $L$ is normal, then $₹$ is interpolative. For if $a^{\prime}<a$ with $y$ as in the definition, then by normality $a^{\prime \prime} \vee y=\top$ for some $a^{\prime \prime}<a$, and then also $a^{\prime}<a^{\prime \prime}$. Another useful fact is that if $a_{i}<a$ $(i=1,2)$, then $a_{1} \vee a_{2}<a$. We find also that the rounded $₹$-ideals of $L$ are exactly the ordinary ideals $I$ (with respect to $\leq$ ) that are rounded in the sense that $I=\downarrow I$.

Proposition 14. Let $L$ be a normal distributive lattice. Then there is a bijection between its regular ideals and its $<$-ideals.

\footnotetext{
${ }^{10}$ Note that the ' $\mathrm{R}$ ' in RIdl stands for rounded, not regular.

11 [40] calls it $\operatorname{Idl}(X,<)$, but we want to stress the roundedness.
} 
Proof. First, if $J$ is a regular ideal then $\downarrow J$ is a $₹$-ideal. The only mildly non-obvious part of this is that if $a_{i}^{\prime} \gtrless a(i=1,2)$, using $y_{i}$ as in the definition, then $a_{1}^{\prime} \vee a_{2}^{\prime} \gtrless a$ using $y_{1} \wedge y_{2}$.

Next, if $I$ is a $<$-ideal then we write

$$
r\langle I\rangle=\{a \in L \mid \downarrow a \subseteq I\} .
$$

To show this is an ideal, suppose $\downarrow a_{i} \subseteq I$ and $b \gtrless a_{1} \vee a_{2}$ with $y$ as in the definition. Then we can find $a_{i}^{\prime} \gtrless a_{i}$ with $a_{1}^{\prime} \vee a_{2}^{\prime} \vee y=\top$ and it follows that $b \gtrless a_{1}^{\prime} \vee a_{2}^{\prime} \in I$. It is regular, because if $\downarrow a \subseteq r\langle I\rangle$ then $\downarrow \downarrow a \subseteq I$; but $\downarrow \downarrow a=\downarrow a$ by interpolativity of $<$, so $a \in r\langle I\rangle$. Indeed, it is the smallest regular ideal containing $I$.

It is now easy to show this gives a bijection, with $r\langle\downarrow J\rangle=J$ and $\downarrow r\langle I\rangle=I$ (using $\downarrow I=I$ ).

We now prove our central result in this section, which gives a geometric account of the opens of $\mathrm{RSpec} L$. One can compare it with Stone's representation theorem for Boolean algebras $B$ (see [29]), where the frame of opens for the spectrum of $B$ is the ideal completion of $B$. In fact, our result generalizes that, since every Boolean algebra is normal, with $₹$ coinciding with $\leq$.

Theorem 15. If $L$ is a normal distributive lattice, then $\operatorname{RIdl} L \cong \mathbb{S}^{\mathrm{RSpec} L}$.

The evaluation map $\operatorname{RIdl} L \times \operatorname{RSpec} L \rightarrow \mathbb{S}$ takes $(I, x)$ to the top point $\top$ whenever the ideal I meets the filter $x$.

Proof. We use [41, Theorem 12.7], which states that if $X$ and $W$ are locales for which $\mathbb{S}^{X}$ exists and is homeomorphic to the double powerlocale $\mathbb{P} W$, then $\mathbb{S}^{W}$ exists and is homeomorphic to $X$. In our case we take $X$ to be RIdl $L$, which is exponentiable with its opens the rounded upsets of $L$, and $W$ to be $\operatorname{RSpec} L$, so it remains to calculate the double powerlocale $\mathbb{P} W$ and show that its points are again the rounded upsets of $L$.

We use the Double Coverage Theorem [38, Theorem 7] to calculate its points. As explained in [44], the calculation is an analogue of one for the lower powerlocale that is directly derived from the usual Coverage Theorem [29]. Given a site in the form of a meet-semilattice $S$ with a meet-stable coverage $\triangleleft$, the corresponding locale has for its points the filters $F$ of $S$ that "split" $\triangleleft$ in the sense that if $a \triangleleft U$ and $a \in F$ then $F$ meets $U$. Then its lower powerlocale has for its points the upsets of $S$ (thus we drop the requirement that meets should be respected) that split $\triangleleft$. For the double powerlocale we require a $D L$-site, in which $S$ is a distributive lattice and the coverage is stable for both meets and joins: the points for the locale are the prime filters that split $\triangleleft$, and for the double powerlocale they are the upsets that split $\triangleleft$.

To apply this theorem we must show that the covers $a \triangleleft \downarrow a$ in $L$ are meet- and join-stable. Meetstability means that $a \wedge b$ is covered by $\left\{a^{\prime} \wedge b \mid a^{\prime}<a\right\}$. It is not true that if $a^{\prime}<a$ then $a^{\prime} \wedge b<a \wedge b$. However, knowing that $a \wedge b \triangleleft \downarrow(a \wedge b)$, we argue that if $c \gtrless a \wedge b$ then $c \gtrless a^{\prime} \gtrless a$ for some $a^{\prime}$, and so $c \leq a^{\prime} \wedge b$. Join-stability is similar, but with joins.

It is clear that an upset splits $\triangleleft$ iff it is rounded with respect to $₹$, and the result follows. The final part derives from the definition of the evaluation map for RIdl $L$.

Now that the notion of commutative $\mathrm{G}^{*}$-algebra has been settled, and the geometricity of the construction of its spectrum has been demonstrated, the following theorem is immediate.

Theorem 16. Let $\underline{A}$ be a unital commutative $C^{*}$-algebra in a topos $\mathscr{E}$ and let $\underline{\Sigma}_{\underline{A}}$ be the Gelfand spectrum of $\underline{A}$. Let $\operatorname{Sh}_{\mathscr{E}}\left(\underline{\Sigma}_{\underline{A}}\right) \rightarrow \mathscr{E}$ be the unique localic geometric morphism corresponding to the locale $\underline{\Sigma}_{\underline{A}}$, and 
let $f: \mathscr{F} \rightarrow \mathscr{E}$ be any geometric morphism. Consider the commutative $G^{*}$-algebra $f^{*}(\underline{A})$, and let $\underline{\Sigma}_{f^{*}(\underline{A})}$ be its spectrum in $\mathscr{F}$. Then we have a pullback square

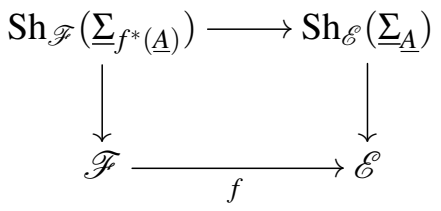

We finish the section by observing that Theorem 15 and the results in [11, 47] provide entirely analoguous results for the geometric theory of integrals on a $\mathrm{G}^{*}$-algebra. Following [22] these internal integrals correspond to quasi-states externally.

\subsection{Computation of the spectrum}

In this subsection we fix a unital $\mathrm{C}^{*}$-algebra $A$ and calculate, in various forms, the spectrum as it arises in the copresheaf approach.

To summarize the notation, $\mathscr{C}(A)$ is the poset of unital commutative $\mathrm{C}^{*}$-subalgebras of $A$, partially ordered by inclusion, and the topos $[\mathscr{C}(A)$, Set $]$ is the category of copresheaves on $\mathscr{C}(A)$, equivalent to the sheaf topos $\operatorname{Sh}(\operatorname{Idl} \mathscr{C}(A))$. $\underline{A}$ is the tautological copresheaf mapping each context $C$ to itself, and mapping each arrow $D \rightarrow C$ in $\mathscr{C}(A)$ to the inclusion $D \hookrightarrow C$. It is a unital commutative $C^{*}$-algebra, internal to the topos $[\mathscr{C}(A)$, Set $]$ and leads - internally - to a normal distributive lattice $\underline{L}_{A}$ and a Gelfand spectrum $\underline{\Sigma}_{A} \cong \underline{\operatorname{RIdl}}\left(\underline{L}_{A}\right)$. This compact regular locale is of interest to the copresheaf model, as it internally plays the role of a phase space.

We seek the external representions of the Gelfand spectrum and other internal locales as bundles, which we shall typically denote by removing underlinings - sometimes in an ad hoc way. The external representation of $\underline{\Sigma}_{\underline{A}}$ will be $p: \Sigma_{\underline{A}} \rightarrow \operatorname{Idl} \mathscr{C}(A)$.

First we calculate the locale $\Sigma_{\underline{A}}$. We characterize both its points (Theorem 17 ), in line with the geometric approach, and its opens (Theorem 19p. We find it convenient to use local compactness to describe the opens geometrically as points of the exponential $\mathbb{S}^{\Sigma_{A}}$, and this is easily translated into a description of the frame $\mathscr{O} \Sigma_{\underline{A}}$.

The explicit description of the opens was previously given Subsection 2.2 of [50]. Although the proof given there has the advantage of not using any advanced topos-theoretic methods, it has some disadvantages too. The proof hides the role of geometric reasoning. As we have seen in the previous subsection, it is because of geometricity that the spectrum is so closely related to the spectra of the commutative $\mathrm{C}^{*}$-subalgebras (or, for reader familiar with the work of Butterfield and Isham, why the spectrum is so closely related to the spectral presheaf of their approach). Another disadvantage of the proof in [50] is that it is not clear how it can be generalized when the topos $[\mathscr{C}(A)$, Set $]$ is replaced by a different topos. This point is also related to the geometricity being hidden.

Theorem 17. The points of $\Sigma_{\underline{A}}$ can be geometrically described as the pairs $(I, x)$ where I is an ideal of $\mathscr{C}(A)$ and $x$ is a subset of $\coprod_{C \in \mathscr{C}(A)} L_{C}$ satisfying the following properties:

1. If $(C, a) \in x$ then $C \in I$.

2. If $C \subseteq D \in I$ then $(C, a) \in x$ iff $\left(D, L_{C D}(a)\right) \in x$.

3. If $C \in I$ then $\left\{a \in L_{C} \mid(C, a) \in x\right\}$ is a regular prime filter in $L_{C}$. 
Proof. Recall from Subsection 2.2 that a point of $\Sigma_{\underline{A}}$ is equivalent to a pair $(I, x)$ where $I$ is an ideal of $\mathscr{C}(A)$ (i.e. a point of the locale $\operatorname{Idl} \mathscr{C}(A)$ ), and $x$ is a point of $I^{*} \Sigma_{\underline{A}}$, in other words, a regular prime filter of $L_{I}$. Now $I$ is the filtered colimit (actually here a directed join) of the principal ideals $\downarrow C$ for $C \in I$, and geometric constructions preserve filtered colimits. It follows that

$$
L_{I} \cong \operatorname{colim}_{C \in I} L_{C}
$$

Each element of $L_{I}$ is the image of some $(C, a)$, so to specify the filter $x$ of $L_{I}$ it suffices to specify $x \subseteq \amalg_{C \in \mathscr{C}(A)} L_{C}$. Then condition (1) says that $x$ is in the colimit over $C \in I$, and (2) expresses the fact that equality in the filtered colimit derives from $(C, a)=\left(D, L_{C D}(a)\right)$. The final condition expresses the regular prime filter property. However, some care is needed with regularity. Actually, regularity says that if $(C, a) \in x$ then there is some $D \in I$ with $C \subseteq D$ and some $b \gtrless L_{C D}(a)$ in $L_{D}$ such that $(D, b) \in x$. Lemma 12 says that in this case there is some $a^{\prime} \in L_{C}$ with $a^{\prime}<a$ and $b \leq L_{C D}\left(a^{\prime}\right)$, and we then have that $\left(C, a^{\prime}\right) \in x$.

Note how this geometric description provides a subbase for the topology (a set of generators for the frame) as the pairs $(C, a)$, where $C \in \mathscr{C}(A)$ and $a \in L_{C}$. The point $(I, x)$ is in this open iff $C \in I$ and $(C, a) \in x$.

We now use local compactness of $\underline{\Sigma}_{\underline{A}}$ to give an explicit description in geometric form of the internal frame, as $\underline{\mathbb{S}}^{\underline{\Sigma_{A}}}$. We shall write $\mathbb{S}^{\underline{\Sigma_{A}}}$ for its external description.

Theorem 18. The points of $\mathbb{S}_{\underline{\underline{E}}}$ are pairs $(I, U)$ where $I$ is an ideal of $\mathscr{C}(A)$ and $U$ is a subset of $\amalg_{C \in \mathscr{C}(A)} L_{C}$ satisfying the following properties.

1. If $(C, a) \in U$ then $C \in I$.

2. If $C \subseteq D \in$ I then $(C, a) \in U$ iff $\left(D, L_{C D}(a)\right) \in U$.

3. If $C \in$ I then $\left\{a \in L_{C} \mid(C, a) \in U\right\}$ is an ideal in $L_{C}$.

4. If $(C, a) \in U$ then there is some $(D, b) \in U$ with $C \subseteq D$ and $L_{C D}(a) \gtrless b$.

Proof. The first part is an application of Theorem 15. The rest is done in exactly the same way as Theorem 17, except we have to take care in expressing the fact that $L_{I}$ is rounded under $₹$, because Lemma 12 does not apply. Note that in the case where $I$ is a principal ideal $\downarrow D, U$ is equivalent to a ₹-ideal of $L_{D}$ and hence to an open of $\Sigma_{D}$.

We can now give an explicit description of the frame $\mathscr{O} \Sigma_{\underline{A}}$.

Theorem 19. $\Sigma_{\underline{A}}$ is locally compact. The points of $\mathbb{S}^{\Sigma_{\underline{A}}}$ are the $\mathscr{C}(A)$-indexed families $U$, where each $U_{C}$ is a $<$-ideal of $L_{C}$, and if $C \subseteq D$, then $L_{C D}\left(U_{C}\right) \subseteq U_{D}$.

Proof. By Theorem 7 we know that $\Sigma_{\underline{A}}$ is locally compact, and that $\mathbb{S}^{\Sigma_{\underline{A}}}$ has the sections of $\mathbb{S}^{\underline{\Sigma}_{\underline{A}}}$ for its points. Since a section is a map from $\operatorname{Idl} \mathscr{C}(A)$, we can use Lemma 2 to see that the sections are as described in the statement.

In terms of frames, it is now immediate that $\mathscr{O} \Sigma_{\underline{A}}$ is isomorphic to the subframe of $\prod_{C \in \mathscr{C}(A)} \mathscr{O} \Sigma_{C}$ comprising those elements $U$ such that for any $C \subseteq D, \rho_{D C}^{*}\left(U_{C}\right) \subseteq U_{D}$.

Unlike the case for the external $\mathbb{S}^{\Sigma_{A}}$, it is a non-trivial calculation to calculate the internal frame $\underline{\mathscr{O}}\left(\underline{\Sigma}_{A}\right)=\operatorname{pt}\left(\underline{\mathbb{S}} \underline{\underline{\Sigma}}_{\underline{A}}\right)$ We should like to emphasize that this non-trivial calculation is usually unnecessary. However much one might like to think that the internal locale "is" the internal frame, it is usually better to identify the locale with its external description. 
Theorem 20. The internal frame $\underline{\mathscr{O}}\left(\underline{\Sigma}_{A}\right)$ is given as a copresheaf by

$$
\underline{\mathscr{O}}\left(\underline{\Sigma}_{A}\right)=\left\{U \in \prod_{C \subseteq D} \mathscr{O} \Sigma_{D} \mid \text { if } C \subseteq D_{1} \subseteq D_{2} \text { then } \rho_{D_{2} D_{1}}^{*}\left(U_{D_{1}}\right) \subseteq U_{D_{2}}\right\} .
$$

Proof. By Yoneda's Lemma,

$$
\underline{\mathscr{O}}\left(\underline{\Sigma}_{A}\right)(C) \cong[\mathscr{C}(A), \operatorname{Set}]\left(\mathscr{Y}(C), \underline{\mathscr{O}}\left(\underline{\Sigma}_{A}\right),\right.
$$

where $\mathscr{Y}: \mathscr{C}(A)^{o p} \rightarrow[\mathscr{C}(A)$, Set $]$ is the Yoneda embedding. Now, internally, $\underline{\mathscr{O}}\left(\underline{\Sigma}_{A}\right)$ is the set of points of $\underline{\mathbb{S}}^{\underline{\Sigma_{A}}}$, in other words its discrete coreflection. It follows that morphisms $\mathscr{Y}(C) \rightarrow \underline{\mathscr{O}}\left(\underline{\Sigma}_{A}\right)$ are equivalent to locale maps

$$
\mathscr{Y}(C) \rightarrow \underline{\mathbb{S}} \underline{\underline{\Sigma}_{A}} \cong \underline{\operatorname{RIdl}}\left(\underline{L_{A}}\right),
$$

and we can analyse these by their external representation. Externally, the local homeomorphism for $\mathscr{Y}(C)$ has bundle space $\operatorname{Idl}(\uparrow C)$. This can be proved by iterated forcing - see Section 2.3. Hence we seek maps $\operatorname{Idl}(\uparrow C) \rightarrow \mathbb{S}^{\Sigma_{A}}$ over $\operatorname{Idl} \mathscr{C}(A)$ and, by Lemma 2, the required maps are the same as monotone families $U_{D}(C \subseteq D)$, with each $U_{D}$ in the fibre of $\mathbb{S}^{\Sigma_{\underline{A}}}$ over $\downarrow D$, in other words a rounded ideal of $L_{D}$. "Monotone" is with respect to the given order on $\mathscr{C}(A)$ and the specialization order on $\mathbb{S}^{\Sigma_{A}}$. Using Proposition 1 and Theorem 13 , we see that if $C \subseteq D_{1} \subseteq D_{2}$, then we require $\operatorname{RIdl}\left(L_{D_{1} D_{2}}\right)\left(U_{D_{1}}\right) \subseteq U_{D_{2}}$. Now suppose $I \subseteq L_{D_{1}}$ is a $₹$-ideal, and $y \subseteq L_{D_{2}}$ a regular prime filter. Then the point $\rho_{D_{2} D_{1}}^{*}(y)=L_{D_{1} D_{2}}^{-1}(y)$ is in the open $I$ iff, as subsets of $L_{D_{1}}$, they meet - they have inhabited intersection. Clearly this is equivalent to $L_{D_{1} D_{2}}(I)$ meeting $y$, which in turn is equivalent to $y$ being in $\operatorname{RIdl}\left(L_{D_{1} D_{2}}\right)(I)=\downarrow L_{D_{1} D_{2}}(I)$. It follows that $\operatorname{RIdl}\left(L_{D_{1} D_{2}}\right)=\rho_{D_{2} D_{1}}^{*}$, and we have the description in the statement.

\section{4 $C^{*}$-algebras in functor categories}

In this subsection we generalize Theorem 19 to unital commutative $\mathrm{C}^{*}$-algebras in toposes that are functor categories. Subsequently, in Subsection 3.5, we use this result when we explore examples of functor categories (other than $[\mathscr{C}(A)$, Set $]$, or $\left[\mathscr{C}(A)^{\mathrm{op}}\right.$, Set $]$ ) which may be of interest to the topos approaches to quantum theory. As a first step, we use presheaf semantics to identify all $\mathrm{C}^{*}$-algebras in functor categories. The reader unfamiliar with presheaf semantics may want to consult [33, Chapter VI].

Let $\mathscr{C}$ be any small category. Below we prove the following:

Proposition 21. The object $\underline{A}$ (with additional structure $\pm,+, \underline{*}, \underline{0}$ ) is a $C^{*}$-algebra in the topos $[\mathscr{C}$, Set $]$ iff it is given by a functor $\underline{A}: \mathscr{C} \rightarrow \mathbf{C S t a r}$, where $\mathbf{C S t a r}$ is the category of $C^{*}$-algebras and $*$-homomorphisms in Set. The $C^{*}$-algebra $\underline{A}$ is commutative iff each $\underline{A}(C)$ is commutative. The algebra $\underline{A}$ is unital iff every $\underline{A}(C)$ is unital and for each $f: C \rightarrow D$, the $*$-homomorphism $\underline{A}(f): \underline{A}(C) \rightarrow \underline{A}(D)$ preserves the unit.

Proof. It follows from Lemma 2 and the discussion in Subsection 3.2 that a semi-normed $*$-algebra over $\underline{\mathbb{C}}$ in $[\mathscr{C}$, Set $]$ is equivalent to a functor $\underline{A}: \mathscr{C} \rightarrow$ Set, such that each $\underline{A}(C)$ is a semi-normed $*$-algebra over $\mathbb{C}$, and, for each arrow $f: D \rightarrow C$ in $\mathscr{C}$, the function $\underline{A}(f): \underline{A}(D) \rightarrow \underline{A}(C)$ is a $*$-homomorphism such that $\|\underline{A}(f)(a)\|_{D} \leq\|a\|_{C}$. We used $\|\cdot\|_{C}$ to denote the semi-norm on $\underline{A}(C)$. The internal semi-norm $\underline{N}$ is submultiplicative and satisfies the $C^{*}$-property iff each semi-norm $\|\cdot\|_{C}$ is submultiplicative and satisfies the $\mathrm{C}^{*}$-property $\left\|a^{*} a\right\|_{C}=\|a\|_{C}^{2}$.

Recall that the semi-norm $\underline{N}$ of $\underline{A}$ is defined as a subobject of $\underline{A} \times \mathbb{Q}^{+}$. The internal semi-norm is connected to the external semi-norms by the identities

$$
\underline{N}(C)=\left\{(a, q) \in \underline{A}(C) \times \mathbb{Q}^{+} \mid\|a\|_{C}<q\right\},
$$




$$
\|\cdot\|_{C}: \underline{A}(C) \rightarrow \mathbb{R}_{0}^{+}, \quad\|a\|_{C}=\inf \left\{q \in \mathbb{Q}^{+} \mid(a, q) \in \underline{N}(C)\right\} .
$$

Note that the fact that $*$-homomorphisms are contractions, in the sense that $\|\underline{A}(f)(a)\|_{C} \leq\|a\|_{C}$, precisely states that $\underline{N}$ defined by $\sqrt{4}$ is a well-defined subobject of $\underline{A} \times \underline{\mathbb{Q}^{+}}$. The semi-norm $\underline{N}$ is a norm iff it satisfies the axiom

$$
\left(\forall q \in \underline{\mathbb{Q}^{+}}(a, q) \in \underline{N}\right) \Rightarrow(a=\underline{0}) .
$$

By the rules of presheaf semantics, externally, this axiom translates to: for each $C \in \mathscr{C}$ the semi-norm $\|\cdot\|_{C}$ is a norm.

Completeness can be checked in the same way as in [21], because the axiom of dependent choice works in any presheaf topos. For completeness, we thus need to check the axiom

$$
\begin{gathered}
\forall f \in \underline{A}^{\mathbb{N}}\left(\left(\forall n \in \underline{\mathbb{N}} \forall m \in \underline{\mathbb{N}}\left(f(n)-f(m), 2^{-n}+2^{-m}\right) \in \underline{N}\right)\right. \\
\left.\Rightarrow\left(\exists a \in \underline{A} \forall n \in \underline{\mathbb{N}}\left(a-f(n), 2^{-n}\right) \in \underline{N}\right)\right) .
\end{gathered}
$$

Note that for any object $C \in \mathscr{C}$, the elements of $\underline{A}^{\mathbb{N}}(C)$ correspond exactly to sequences $\left(a_{n}\right)_{n \in \mathbb{N}}$ in $\underline{A}(C)$. By presheaf semantics, the axiom for completeness holds iff for every object $C \in \mathscr{C}$ and any sequence $\left(a_{n}\right)_{n \in \mathbb{N}}$ in $\underline{A}(C)$, if

$$
C \Vdash\left(\forall n \in \underline{\mathbb{N}} \forall m \in \underline{\mathbb{N}}\left(a_{n}-a_{m}, 2^{-n}+2^{-m}\right) \in \underline{N}\right),
$$

then

$$
C \Vdash\left(\exists a \in \underline{A} \forall n \in \underline{\mathbb{N}}\left(a-a_{n}, 2^{-n}\right) \in \underline{N}\right) .
$$

This can be simplified by repeated use of presheaf semantics, and the identity

$$
\left\|\underline{A}(f)\left(a_{n}\right)-\underline{A}(f)\left(a_{m}\right)\right\|_{D}=\left\|\underline{A}(f)\left(a_{n}-a_{m}\right)\right\|_{D} \leq\left\|a_{n}-a_{m}\right\|_{C}
$$

where $f: C \rightarrow D$ is any arrow. In the end, the axiom of completeness simplifies to the statement that given an object $C \in \mathscr{C}$ and any sequence $\left(a_{n}\right)_{n \in \mathbb{N}}$ in $\underline{A}(C)$ such that for any pair $n, m \in \mathbb{N}$ we have $\left(a_{n}-a_{m}, 2^{-n}+2^{-m}\right) \in \underline{N}(C)$, there exists an element $a \in \underline{A}(C)$ such that for every $n \in \mathbb{N},\left(a-a_{n}, 2^{-n}\right) \in$ $\underline{N}(C)$. By definition of $\underline{N}$ this simply states that each $\underline{A}(C)$ is complete with respect to the norm $\|\cdot\|_{C}$. This completes the proof that $\mathrm{C}^{*}$-algebras in $[\mathscr{C}$, Set $]$ are equivalent to functors $\mathscr{C} \rightarrow \mathbf{C S t a r}$.

Proposition 21 makes it easy to calculate the spectrum $\underline{\Sigma}_{A}$ of any commutative unital $\mathrm{C}^{*}$-algebra $\underline{A}$ in any functor category, using the methods of Subsection 3.3. The first step is the construction of the distributive lattice $\underline{L}_{A}$, which we will simply denote as $\underline{L}$. This is done as in Subsection 3.2. The construction is geometric, based on the $\mathrm{G}^{*}$-algebra structure of $\underline{A}$, and so from the discussion in Section 2 we can derive $\underline{L}$ :

$$
\begin{gathered}
\underline{L}: \mathscr{C} \rightarrow \text { Set, } \underline{L}(C)=L_{\underline{A}(C)}, \\
\underline{L}(f)=L_{\underline{A}(f)}: L_{\underline{A}(C)} \rightarrow L_{\underline{A}(D)} .
\end{gathered}
$$

As in Subsection 3.3, we can now describe the spectrum $\Sigma$ in various ways. To start with, it is described externally as a localic geometric morphism $\Sigma \rightarrow[\mathscr{C}$, Set $]$. We must keep in mind that if $\mathscr{C}$ is not a poset, then neither $\Sigma_{\underline{A}}$ nor $[\mathscr{C}$, Set $]$ is a localic topos: they classify non-propositional theories and are generalized spaces in the sense of Grothendieck. However, the bundle $\Sigma_{\underline{A}} \rightarrow[\mathscr{C}$, Set $]$ is still a localic bundle, because it is a localic geometric morphism [30, A4.6.1]. Its fibres are locales. This all follows because it arises from an internal locale in $[\mathscr{C}$, Set $]$. 
To make clear the analogy with the localic case, we shall use the same notation for a locale as for its topos of sheaves. For example, 1 will often denote the topos of sets, and $\mathbb{S}$ the topos $\mathrm{Sh} \mathbb{S}$, whose objects are functions. In practice this does not cause confusion, but one should refrain from asking whether the locale actually "is" the frame or the topos. Rather, one should think that a locale is described equally well by its frame of opens or its topos of sheaves. Note that geometric morphisms between localic toposes are equivalent to maps between the locales, and we shall frequently refer to geometric morphisms in general as maps between the toposes.

For the rest of this subsection we shall let $\mathscr{C}$ be a small category, and let $\underline{A}$ be a $\mathrm{C}^{*}$-algebra in $[\mathscr{C}$, Set $]$. We write $p: \Sigma_{\underline{A}} \rightarrow[\mathscr{C}$, Set $]$ for the localic bundle corresponding to the internal spectrum $\underline{\Sigma}_{A}$.

Theorem 22. (Analogue of Theorem 17) The points of $\Sigma_{A}$ can be geometrically described as the pairs $(F, x)$ where $F$ is a flat presheaf over $\mathscr{C}$ and $x$ is a subset of $\amalg_{(C, u) \in\left(\int F\right)_{0}} L_{C}$ satisfying the following properties:

1. If $f:(C, u) \rightarrow(D, v)$ in $\int F$ then $(C, u, a) \in x$ iff $\left(D, v, L_{f}(a)\right) \in x$.

2. If $(C, u)$ is an object in $\int F$ then $\left\{a \in L_{C} \mid(C, u, a) \in x\right\}$ is a regular prime filter in $L_{C}$.

Proof. The proof is essentially the same as that of Theorem 17, once one has taken on the fact (Subsection 2.3 , just below Definition 3 that the points of $[\mathscr{C}$, Set $]$ are the flat presheaves over $\mathscr{C}$ and that each is a filtered colimit of representables $\mathscr{Y}(C)$.

Theorem 23. (Analogue of Theorem 18) The frame of opens of $\underline{\Sigma}_{A}$ has internal localic form $\mathbb{S}_{\underline{\underline{S}}}^{\underline{\Sigma}_{A}}=$ $\underline{\operatorname{RId}}\left(\underline{L}_{A}\right)$. Externally, the points of the bundle locale $\mathbb{S}^{\underline{\Sigma}_{A}}$ are pairs $(F, U)$, where $F$ is a flat presheaf of $\mathscr{C}$ and $U$ is a subset of $\amalg_{(C, u) \in\left(\int F\right)_{0}} L_{C}$ satisfying the following properties:

1. If $f:(C, u) \rightarrow(D, v)$ in $\int F$, then $(C, u, a) \in U$ iff $\left(D, v, L_{f}(a)\right) \in U$.

2. If $(C, u)$ is an object in $\int F$, then $\left\{a \in L_{C} \mid(C, u, a) \in U\right\}$ is an ideal in $L_{C}$.

3. If $(C, u, a) \in U$, then there is some $f:(C, u) \rightarrow(D, v)$ in $\int F$ and some $(D, v, b) \in U$ with $L_{f}(a) \gtrless b$.

Proof. Essentially the same as for Theorem 18 .

At this point in Subsection 3.3 we moved on to giving an explicit description of the frame $\mathscr{O} \Sigma_{A}$. Here we must be more careful, since in general $\Sigma_{A}$ is a non-localic topos. Any topos map (geometric morphism) has a hyperconnected-localic factorization [30, A4.6.1], and by applying this to a map $\mathscr{E} \rightarrow 1$ we see that $\mathscr{E}$ has a localic reflection $\mathscr{L}(\mathscr{E})$. Its opens are the maps $\mathscr{E} \rightarrow \mathbb{S}$. In other words, since $\mathbb{S}$ classifies subsingletons, the opens of $\mathscr{L}(\mathscr{E})$ are the subobjects of 1 in $\mathscr{E}$. If $\mathscr{E}$ is exponentiable as a topos (as is the case for $[\mathscr{C}$, Set $]$ ), then the opens of $\mathscr{L}(\mathscr{E})$ are the points of $\mathbb{S}^{\mathscr{E}}$ and can be calculated immediately by the method of Theorem 19 .

Theorem 24. (Analogue of Theorem 19) The opens of $\Sigma_{A}$ (equivalently: the opens of the localic reflection $\mathscr{L}\left(\Sigma_{\underline{A}}\right)$ ) can be geometrically described as the elements $U \in \prod_{C \in \mathscr{C}_{0}} \Sigma_{\underline{A}(C)}$ such that if $f: C \rightarrow D$ is a morphism in $\mathscr{C}$, then $\Sigma_{\underline{A}(f)}^{*}\left(U_{C}\right) \leq U_{D}$.

Proof. Using exponentiability as in Theorem 19, we can calculate the opens as the points of $\mathbb{S}^{\Sigma_{A}}$ and thence as the sections of $\mathbb{S}_{\underline{\underline{E}}} \rightarrow[\mathscr{C}$, Set $]$. Sections are described using Lemma2 2 . For each object $C$ of $\mathscr{C}$ we require a $₹$-ideal of $L_{\underline{A}(C)}$, hence an element of $\mathscr{O} \Sigma_{\underline{A}(C)}$. For each morphism $f: C \rightarrow D$ we require a homomorphism of points of $\mathbb{S}^{\Sigma_{A}}$ over $f$, and the homomorphism property comes down to the condition $\Sigma_{\underline{A}(f)}^{*}\left(U_{C}\right) \leq U_{D}$. 
For completeness, we sketch how one might describe the full topos $\left.\operatorname{Sh}\left(\Sigma_{\underline{A}}\right)\right)$, i.e. the sheaves of $\Sigma_{\underline{A}}$ rather than just its opens (which are not enough, and only describe the localic reflection). Again one would use exponentiability, but this time to calculate $[\mathrm{set}]^{\Sigma}$ instead of $\mathbb{S}^{\Sigma}$. Here, [set] denotes the object classifier, classifying the geometric theory with one sort and no other ingredients. Its models are just sets (carrying the single sort), so the maps $\Sigma \rightarrow$ [set] are the sheaves of $\Sigma$ (by which we just mean the objects of the topos, even in this non-localic case). [49] shows how to give a geometric description of the sheaves over any stably compact locale when presented by a strong proximity lattice, and as a special case works for a compact regular locale when presented by a normal distributive lattice $L$. The sheaves are, first of all, finitary sheaves over the distributive lattice $L$, i.e. those presheaves $F$ that satisfy finite instances of the sheaf pasting condition. In addition, they must satisfy a continuity condition that $F(a) \cong \operatorname{colim}_{a \gtrless a^{\prime}} F\left(a^{\prime}\right)$.

We now proceed by analogy with Theorem 23 , but replacing $\mathbb{S}$ by [set]. Internally in $[\mathscr{C}$, Set] we have a geometric theory describing the sheaves over the internal locale $\underline{\Sigma}_{A}$. This corresponds externally to a bundle (not localic) [set $]_{\underline{\underline{A}}}^{\underline{\Sigma}_{1}} \rightarrow[\mathscr{C}$, Set $]$. We can now describe the points of the external topos $[$ set $] \underline{\underline{\Sigma}}_{\underline{A}}$ as pairs $(F, G)$ where $F$ is a flat presheaf over $\mathscr{C}$ and $G$ is a sheaf (presheaf with finitary pasting and continuity) over $\operatorname{colim}_{(C, u) \in\left(\int F\right)_{0}} L_{\underline{A}(C)}$. After this, the sheaves of $\Sigma$ can be described as the sections of $[\text { set }]_{\underline{\underline{A}}}^{\underline{\Sigma}} \rightarrow[\mathscr{C}$, Set $]$.

Finally in this subsection, we calculate the internal frame of $\underline{\Sigma}_{A}$.

Theorem 25. (Analogue of Theorem 20) The internal frame $\underline{\mathscr{O}}\left(\underline{\Sigma}_{A}\right)$ is given as an object of $[\mathscr{C}$, Set $]$ by

$$
\underline{\mathscr{O}}\left(\underline{\Sigma}_{\underline{A}}\right)(C)=\left\{U \in \prod_{f: C \rightarrow D} \mathscr{O} \Sigma_{D} \mid \text { if } C \stackrel{f}{\rightarrow} D_{1} \stackrel{g}{\rightarrow} D_{2} \text { then } \rho_{g}^{*}\left(U_{f}\right) \leq U_{g f}\right\}
$$

Proof. Just as in Theorem 20, we find that $\underline{\mathscr{O}}\left(\underline{\Sigma}_{A}\right)(C)$ is the set of maps (geometric morphisms) from $\mathscr{Y}(C)$ to $\operatorname{RIdl} L_{A}$ over $[\mathscr{C}$, Set $]$. We can calculate the external form of the discrete locale $\mathscr{Y}(C)$ using iterated forcing (Subsection 2.3). Its topos is $[\mathscr{C} \ltimes \mathscr{Y}(C)$, Set $]$, where now $\mathscr{Y}(C)$ denotes the discrete internal category. From the definition of $\mathscr{Y}$ we see that the objects of $\mathscr{C} \ltimes \mathscr{Y}(C)$ are the pairs $(D, f)$ with $f: C \rightarrow D$ in $\mathscr{C}$, and a morphism from $\left(D_{1}, f_{1}\right)$ to $\left(D_{2}, f_{2}\right)$ is a morphism $g: D_{1} \rightarrow D_{2}$ in $\mathscr{C}$ such that $f_{2}=g f_{1}$.

Now combining Lemma 2 with Theorem 23 , we see that the maps we are looking for are functors $U$ from $\mathscr{C} \ltimes \mathscr{Y}(C)$ to the models as described in Theorem 23 . For each object $f: C \rightarrow D$, its image $U_{f}$ must be such that the flat presheaf $F$ as described in the Theorem is representable (for $D$ ). Its representability allows us to combine conditions (2) and (3) to say that we have a ₹-ideal in $L_{A(D)}$, hence an open of $\Sigma_{A(D)}$. Now for each morphism $g: D \rightarrow D^{\prime}$, with $f^{\prime}=g f$, we need a homomorphism $U_{f} \rightarrow U_{f^{\prime}}$ over $g$. In other words, if $a \in U_{f}$ (as ₹-ideal of $L_{\underline{A}(D)}$ ), then $L_{\underline{A}(g)}(a) \in U_{f^{\prime}}$. When the ₹-ideals of $L_{\underline{A}(D)}$ are viewed as opens of $\Sigma_{\underline{A}(D)}$, this is equivalent to saying that $U_{f} \leq \rho_{\underline{A}(g)}^{*}\left(U_{f^{\prime}}\right)$. We now see that the internal frame is as described.

\subsection{Algebraic quantum field theory}

The copresheaf approach is based on algebraic quantum theory in the sense that in this approach quantum theory is described using abstract $C^{*}$-algebras. In this section we seek to extend the copresheaf approach, as already suggested in [21], to the Haag-Kastler formalism, which is an algebraic approach to quantum field theory. Introductions to the Haag-Kastler formalism, or algebraic quantum field theory (AQFT), can be found in [1,17]. In this formalism (where, for the sake of simplicity we consider Minkowski spacetime $\mathscr{M})$ the physical content of a quantum field theory is described by a net of $\mathrm{C}^{*}$-algebras $O \rightarrow \mathfrak{A}(O)$, 
where $O$ ranges over certain (open connected causally complete) regions of spacetime. This means that we associate to each region $O$ of spacetime of interest, a $\mathrm{C}^{*}$-algebra $\mathfrak{A}(O)$. We think of the self-adjoint elements of $\mathfrak{A}(O)$ as the observables that can be measured in the region $O$. With this in mind, we can make the assumption that if $O_{1} \subseteq O_{2}$, then $\mathfrak{A}\left(O_{1}\right) \subseteq \mathfrak{A}\left(O_{2}\right)$. If $\mathscr{K}(\mathscr{M})$ denotes the set of the spacetime regions of interest, partially ordered by inclusion, then a net of $\mathrm{C}^{*}$-algebras defines a covariant functor $\mathfrak{A}: \mathscr{K}(\mathscr{M}) \rightarrow$ CStar. We assume that the algebras $\mathfrak{A}(O)$ are unital for convenience.

By Proposition 21 an AQFT is a $\mathrm{C}^{*}$-algebra $\mathfrak{A}$ internal to $[\mathscr{K}(\mathscr{M})$, Set $]$. Note that $\mathfrak{A}$ is in general not commutative. As for the copresheaf approach we can Bohrify the $\mathrm{C}^{*}$-algebra $\mathfrak{A}$. This means that we make it commutative by considering it as a copresheaf over the poset of commutative subalgebras. The difference with the copresheaf approach is that the Bohrification takes place internal in the topos $[\mathscr{K}(\mathscr{M})$, Set $]$, instead of the topos Set. We obtain a commutative $\mathrm{C}^{*}$-algebra internal to a topos (which in turn is internal to a functor category), and, using the ideas of the preceding paragraphs, we describe the points of the Gelfand spectrum of this commutative $\mathrm{C}^{*}$-algebra.

Instead of the Haag-Kastler formalism, we could have considered the more general and more recent locally covariant quantum field theories [5]. This amounts to replacing the poset $\mathscr{K}(\mathscr{M})$ by a more complicated category of manifolds and embeddings (which is no longer a poset). Although Bohrification of the locally covariant field theories can be described using the same ideas of Subsection 3.4, we stick with the Haag-Kastler formalism, as this makes the presentation a bit easier.

An internal unital commutative $C^{*}$-subalgebra of $\mathfrak{A}$ is simply a subobject $\mathfrak{C}$ of $\mathfrak{A}$ such that for each $O \in \mathscr{K}(\mathscr{M}), \mathfrak{C}(O)$ is a commutative unital $\mathrm{C}^{*}$-algebra in Set. These internally defined commutative $\mathrm{C}^{*}$-subalgebras form a poset $\mathscr{C}(\mathfrak{A})$ in $[\mathscr{K}(\mathscr{M})$, Set $]$ and we can consider the (internal) functor category over this poset. By using iterated forcing (see Section 2.3, we can describe this functor category within a functor category using a single Grothendieck topos over Set, given by the site $\mathscr{K}(\mathscr{M}) \ltimes \mathscr{C}(\mathfrak{A})$. In this (composite) topos, the Bohrified net is given by the functor $(O, \mathfrak{C}) \mapsto \mathfrak{C}(O)$, where $O \in \mathscr{K}(\mathscr{M})$ and $\mathfrak{C}$ is a commutative unital $\mathrm{C}^{*}$-subalgebra of $\left.\mathfrak{A}\right|_{\uparrow O}$. Before considering Gelfand Duality, we first simplify the topos in which we are working. Instead of labeling the objects of the base category by subalgebras $\mathfrak{C}$ of $\left.\mathfrak{A}\right|_{\uparrow O}$, we only concentrate on the part $\mathfrak{C}(O)$. This motivates using the topos $[\mathscr{P}$,Set $]$, where the poset $\mathscr{P}$ is defined as follows: an element $(O, C) \in \mathscr{P}$, consists of an $O \in \mathscr{K}(\mathscr{M})$ and a $C \in \mathscr{C}_{O}:=\mathscr{C}(\mathfrak{A}(O))$, and the order relation is given by

$$
\left(O_{1}, C_{1}\right) \leq\left(O_{2}, C_{2}\right) \text { iff } O_{1} \subseteq O_{2}, C_{1} \subseteq C_{2} .
$$

We are interested in the unital commutative $\mathrm{C}^{*}$-algebra $\underline{A}: \mathscr{P} \rightarrow$ Set, $(O, C) \mapsto C$ in the topos $[\mathscr{P}$, Set $]$. Note that $\underline{A}\left(\left(O_{1}, C_{1}\right) \leq\left(O_{2}, C_{2}\right)\right)$ is the inclusion map $C_{1} \hookrightarrow C_{2}$.

Next, we want to compute the points of the locale $\Sigma$, which is the external description of the Gelfand

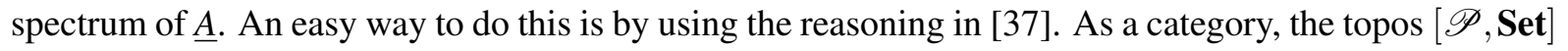
is equivalent to the topos $\operatorname{Sh}\left(\mathscr{P}_{\uparrow}\right)$, where $\mathscr{P}_{\uparrow}$ is the set $\mathscr{P}$, equipped with the Alexandrov upset topology. As in [34] we can find a site $\mathscr{P} \ltimes \underline{\Sigma}$ such that $\operatorname{Sh}_{\mathrm{Sh}(\mathscr{P})}(\underline{\Sigma})$, the topos of sheaves over $\underline{\Sigma}$, internal to $\operatorname{Sh}\left(\mathscr{P}_{\uparrow}\right)$ is equivalent to $\operatorname{Sh}(\mathscr{P} \ltimes \Sigma)$. The locale $\Sigma$ is the locale generated by the posite $\mathscr{P} \ltimes \Sigma$. We use the posite description $\mathscr{P} \ltimes \underline{\Sigma}$ in order to find the points. We start with the functor $\underline{L}$, the distributive lattice object in $[\mathscr{P}$, Set $]$, given by

$$
\begin{gathered}
\underline{L}: \mathscr{P} \rightarrow \text { Set }, \underline{L}(O, C)=L_{C}, \\
\underline{L}\left(\left(O_{1}, C_{1}\right) \leq\left(O_{2}, C_{2}\right)\right): L_{C_{1}} \rightarrow L_{C_{2}},[a]_{C_{1}} \mapsto[a]_{C_{2}} .
\end{gathered}
$$

The elements of $\mathscr{P} \ltimes \underline{\Sigma}$ are triples $\left(O, C,[a]_{C}\right)$, where $O \in \mathscr{K}(\mathscr{M}), C \in \mathscr{C}_{O}$ and $[a]_{C} \in L_{C}$. The order of this poset is given by

$$
\left(O_{1}, C_{1},\left[a_{1}\right]_{C_{1}}\right) \leq\left(O_{2}, C_{2},\left[a_{2}\right]_{C_{2}}\right), \quad \text { iff } O_{2} \subseteq O_{1}, C_{2} \subseteq C_{1},\left[a_{1}\right]_{C_{1}} \leq\left[a_{2}\right]_{C_{1}} .
$$


The poset $\mathscr{P} \ltimes \Sigma$ is equipped with the following covering relation $\triangleleft$, which is inherited from the covering relation $\unlhd$, exploiting the fact that we are working over $\mathscr{P}$. We have a covering $\left(O, C,[a]_{C}\right) \triangleleft W$ iff for $W_{0}=\left\{[b]_{C} \in L_{C} \mid\left(O, C,[b]_{C}\right) \in W\right\}$, the condition $[a]_{C} \triangleleft W_{0}$ holds in $L_{C}$. Note that the covering relation on $\mathscr{P} \ltimes \Sigma$ is completely described in terms of covering relations on the $L_{C}$.

A point $\sigma$ of the external spectrum $\Sigma$ corresponds to a completely prime filter of $\mathscr{P} \ltimes \Sigma$. Recall that a filter $\sigma$ is a nonempty, upward closed and lower directed subset of $\mathscr{P} \ltimes \Sigma$, and that $\sigma$ is completely prime if it satisfies

$$
\left(O, C,[a]_{C}\right) \in \sigma \text { and }\left(O, C,[a]_{C}\right) \triangleleft W \text {, implies } U \cap \sigma \neq \emptyset \text {. }
$$

Let $\sigma$ be a point of $\Sigma$. It is straightforward to show that

$$
\mathscr{R}=\left\{O \in \mathscr{K}(\mathscr{M}) \mid \exists C \in \mathscr{C}_{O}, \exists[a]_{C} \in L_{C}, \text { s.t. }\left(O, C,[a]_{C}\right) \in \sigma\right\}
$$

is an ideal of $\mathscr{K}(\mathscr{M})$. Fix any $O \in \mathscr{R}$ and consider the set

$$
\mathscr{I}_{O}=\left\{C \in \mathscr{C}_{O} \mid \exists[a]_{C} \in L_{C} \text { s.t. }\left(O, C,[a]_{C}\right) \in \sigma\right\} .
$$

For any $O \in \mathscr{R}, \mathscr{I}_{O}$ is an ideal of $\mathscr{C}_{O}$. For a pair $O \in \mathscr{R}$ and $C \in \mathscr{I}_{O}$, define

$$
\sigma_{O, C}:=\left\{[a]_{C} \in L_{C} \mid\left(O, C,[a]_{C}\right) \in \sigma\right\}
$$

As in [37], it can be shown that $\sigma_{O, C}$ is a completely prime filter of $L_{C}$. A completely prime filter $\sigma_{O, C}$ on $L_{C}$ corresponds to a unique point $\lambda(O, C)$ of the Gelfand spectrum $\Sigma_{C}$.

Next, we show how for different $O \in \mathscr{R}, C \in \mathscr{I}_{O}$, the $\lambda(O, C) \in \Sigma_{C}$ are related. Let, for some fixed $O \in \mathscr{R}, D \subset C$ in $\mathscr{C}_{O}$. Let $a \in D^{+}$and assume that $[a]_{C} \in \sigma_{O, C}$. By the order on $\mathscr{P} \ltimes \underline{\Sigma}$,

$$
\left(O, C,[a]_{C}\right) \leq\left(O, D,[a]_{D}\right) \in \sigma
$$

where we used that $\sigma$ is a filter, and therefore it is upward closed. For any $a \in D^{+}$, if $[a]_{C} \in \sigma_{O, C}$, then $[a]_{D} \in \sigma_{O, D}$. The filter $\sigma_{O, D}$ can be viewed as a frame map $\sigma_{O, D}: \mathscr{O} \Sigma_{C} \rightarrow \underline{2}$ mapping the open $X_{a}^{D}=\left\{\lambda \in \Sigma_{D} \mid\langle\lambda, a\rangle>0\right\}$ to 1 iff $\lambda(O, D) \in X_{a}^{D}$, iff $[a]_{D} \in \sigma_{O, D}$. If $\rho_{C D}: \Sigma_{C} \rightarrow \Sigma_{D}$ is the restriction map, then $\sigma_{O, C} \circ \rho_{C D}^{-1}: \mathscr{O} \Sigma_{D} \rightarrow 2$ corresponds to the point $\left.\lambda(O, C)\right|_{D}$. At the level of points of $\Sigma_{D}$, the implication

$$
\forall a \in D^{+}, \quad[a]_{C} \in \sigma_{O, C} \Rightarrow[a]_{D} \in \sigma_{O, D}
$$

translates to:

$$
\forall a \in D^{+}, \quad\left(\sigma_{O, C}\left(X_{a}^{C}\right)=1\right) \Rightarrow\left(\sigma_{O, D}\left(X_{a}^{D}\right)=1\right) .
$$

As the $X_{a}^{D}$ form a basis of the Hausdorff space $\Sigma_{D}$, and $\rho^{-1}\left(X_{a}^{D}\right)=X_{a}^{C}$, this can only mean that $\sigma_{O, D}=$ $\sigma_{O, C} \circ \rho_{D C}^{-1}$. In other words, whenever $D \subseteq C$, one has $\lambda(O, D)=\left.\lambda(O, C)\right|_{D}$.

Assume that $O^{\prime} \subset O$ in $\mathscr{K}(\mathscr{M})$ and that $C \in \mathscr{C}_{O^{\prime}}$. In $\mathscr{P} \ltimes \underline{\Sigma}$,

$$
\forall[a]_{C} \in L_{C}, \quad\left(O, C,[a]_{C}\right) \leq\left(O^{\prime}, C,[a]_{C}\right) .
$$

If $[a]_{C} \in \sigma_{O, C}$, then by the filter property of $\sigma,[a]_{C} \in \sigma_{O^{\prime}, C}$. We conclude that if $O^{\prime} \subseteq O$ in $\mathscr{R}$ and $C \in \mathscr{C}_{O^{\prime}}$, then $\lambda\left(O^{\prime}, C\right)=\lambda(O, C)$. Hence:

Theorem 26. A point $\sigma$ of $\Sigma$ is described by a triple $\left(\mathscr{R}, \mathscr{I}_{\mathscr{R}}, \lambda_{\mathscr{R}, \mathscr{I}}\right)$, where:

- $\mathscr{R}$ is an ideal in $\mathscr{K}(\mathscr{M})$. 
- The function $\mathscr{I}_{\mathscr{R}}$ associates to each $O \in \mathscr{R}$, an ideal $\mathscr{I}_{O}$ of $\mathscr{C}_{O}$ satisfying two conditions. Firstly, if $O_{1} \subseteq O_{2}$, then $\mathscr{I}_{O_{2}} \cap \mathscr{C}_{O_{1}} \subseteq \mathscr{I}_{O_{1}}$. Secondly, if $C_{i} \in \mathscr{I}_{O_{i}}$, where $i \in\{1,2\}$, then there is an $O \in \mathscr{R}$ and $a C \in \mathscr{I}_{O}$ such that $O_{i} \subseteq O$ and $C_{i} \subseteq C$.

- The function $\lambda_{\mathscr{R}, \mathscr{I}}$ associates to each $O \in \mathscr{R}$ and $C \in \mathscr{I}_{O}$, an element $\lambda_{O, C} \in \Sigma_{C}$, such that if $O_{1} \subseteq O_{2}$ and $C_{1} \subseteq C_{2}$, then $\lambda_{O_{1}, C_{1}}=\left.\lambda_{O_{2}, C_{2}}\right|_{C_{1}}$.

The two conditions in the second bullet point are included to ensure that the set

$$
\mathscr{I}=\left\{(O, C) \in \mathscr{P} \mid O \in \mathscr{R}, C \in \mathscr{I}_{O}\right\}
$$

is an ideal of $\mathscr{P}$. Mathematically, the theorem would look more elegant if it were formulated in terms of ideals of $\mathscr{P}$ instead of using pairs $\left(\mathscr{R}, \mathscr{I}_{\mathscr{R}}\right)$, but that description would miss an important physical point. Namely, a spacetime point $x \in M$ corresponds to a specific filter of $\mathscr{K}(\mathscr{M})$, consisting of all $O \in \mathscr{K}(\mathscr{M})$ containing $x$. However, a point $\sigma$ of $\Sigma$ is labelled by an ideal $\mathscr{R}$ of $\mathscr{K}(\mathscr{M})$ and not by a filter. If we want the points of the phase space to be indexed by points of the spacetime $\mathscr{M}$, it might be interesting to look at the contravariant functor $\Sigma: \mathscr{P}^{o p} \rightarrow$ Set, $(O, C) \mapsto \Sigma_{C}$. Remaining on the topic of applying topos approaches to quantum theory to algebraic quantum field theory, this functor $\underline{\Sigma}$ is also interesting when we consider the work by Nuiten on this subject [35]. Nuiten investigates relations between independence conditions on nets of operator algebras on the one hand, and sheaf conditions on the corresponding Bohrified functors on the other. As argued in [51], the sheaf condition of Nuiten can be viewed as a sheaf condition on the functor $\underline{\Sigma}: \mathscr{P}^{o p} \rightarrow$ Set (although strictly speaking the covering relation which is involved does not satisfy all conditions for the basis of a Grothendieck topology).

\section{Acknowledgements}

The authors would like to thank Klaas Landsman for his comments, which greatly improved this paper, and also an anonymous referee for his or her comments.

\section{References}

[1] Huzihiro Araki (1999): Mathematical theory of quantum fields. International Series of Monographs on Physics 101, Oxford University Press, New York.

[2] Bernhard Banaschewski \& Christopher J. Mulvey (2000): The spectral theory of commutative $C^{*}$ algebras: the constructive Gelfand-Mazur theorem. Quaestiones Mathematicae 23(4), pp. 465-488, doi $10.2989 / 16073600009485990$

[3] Bernhard Banaschewski \& Christopher J. Mulvey (2000): The spectral theory of commutative $C^{*}$-algebras: the constructive spectrum. Quaestiones Mathematicae 23(4), pp. 425-464, doi: $10.2989 / 16073600009485989$

[4] Bernhard Banaschewski \& Christopher J. Mulvey (2006): A globalisation of the Gelfand duality theorem. Annals of Pure and Applied Logic 137(1-3), pp. 62-103, doi:10.1016/j.apal.2005.05.018

[5] Romeo Brunetti, Klaus Fredenhagen \& Rainer Verch (2003): The generally covariant locality principle - $a$ new paradigm for local quantum field theory. Comm. Math. Phys. 237(1-2), pp. 31-68, doi 10.1007/s00220003-0815-7. Dedicated to Rudolf Haag.

[6] Jeremy Butterfield \& Chris J. Isham (1999): A topos perspective on the Kochen-Specker theorem: II. Conceptual Aspects and Classical Analogues. International Journal of Theoretical Physics 38(3), pp. 827-859, doi 10.1023/A:1026652817988. 
[7] Jeremy Butterfield \& Chris J. Isham (2002): A topos perspective on the Kochen-Specker theorem: IV. Interval Valuations. International Journal of Theoretical Physics 41(4), pp. 613-639, doi:10.1023/A:1015276209768.

[8] Martijn Caspers, Chris Heunen, Nicolaas P. Landsman \& Bas Spitters (2009): Intuitionistic Quantum Logic of an n-level System. Foundations of Physics 39, pp. 731-759, doi:10.1007/s10701-009-9308-7

[9] Thierry Coquand (2005): About Stone's Notion of Spectrum. Journal of Pure and Applied Algebra 197, pp. 141-158, doi:10.1016/j.jpaa.2004.08.024

[10] Thierry Coquand \& Bas Spitters (2009): Constructive Gelfand duality for $C^{*}$-algebras. Mathematical Proceedings of the Cambridge Philosophical Society 147(2), pp. 339-344, doi $10.1017 /$ S0305004109002515. ArXiv:0808.1518.

[11] Thierry Coquand \& Bas Spitters (2009): Integrals and Valuations. Journal of Logic and Analysis 1(3), pp. 1-22, doi:10.4115/jla.2009.1.3

[12] Andreas Döring (2012): Flows on Generalised Gelfand Spectra of Nonabelian Unital C*-Algebras and Time Evolution of Quantum Systems. Available at http://arxiv.org/abs/1212.4882

[13] Andreas Döring (2012): Generalised Gelfand Spectra of Nonabelian Unital $C^{*}$-Algebras. Available at http: //arxiv.org/abs/1212.2613

[14] Andreas Döring \& John Harding (2010): Abelian subalgebras and the Jordan structure of a von Neumann algebra. Available at http://arxiv.org/abs/1009.4945

[15] Andreas Döring \& Chris Isham (2011): 'What is a Thing?': Topos Theory in the Foundations of Physics. In Bob Coecke, editor: New Structures in Physics, chapter 13, Lecture Notes in Physics 813, Springer, pp. 753-940, doi:10.1007/978-3-642-12821-9_13. ArXiv:0803.0417.

[16] Bertfried Fauser, Guillaume Raynaud \& Steven Vickers (2012): The Born Rule as Structure of Spectral Bundles. In Bart Jacobs, Peter Selinger \& Bas Spitters, editors: Proceedings of the 8th International Workshop on Quantum Physics and Logic, Nijmegen 2011, EPTCS 95, pp. 81-90, doi:10.4204/EPTCS.95.8.

[17] Rudolf Haag (1996): Local Quantum Physics: Fields, Particles, Algebras, second edition. Texts and Monographs in Physics, Springer-Verlag, Berlin, doi:10.1007/978-3-642-61458-3

[18] Jan Hamhalter (2011): Isomorphisms of ordered structures of abelian $C^{*}$-subalgebras of $C^{*}$-algebras. J. Math. Anal. Appl. 383, pp. 391-399, doi:10.1016/j.jmaa.2011.05.035

[19] Jan Hamhalter \& Ekaterina Turilova (2013): Structure of associative subalgebras of Jordan operator algebras. The Quarterly Journal of Mathematics 64(2), pp. 397-408, doi:10.1093/qmath/has015

[20] John Hamilton, Chris J. Isham \& Jeremy Butterfield (2000): A topos perspective on the Kochen-Specker theorem: III. Von Neumann Algebras as the Base Category. International Journal of Theoretical Physics 39(6), pp. 1413-1436, doi 10.1023/A:1003667607842.

[21] Chris Heunen, Klaas Landsman \& Bas Spitters (2009): A topos for algebraic quantum theory. Communications in mathematical physics 291(1), pp. 63-110, doi:10.1007/s00220-009-0865-6. ArXiv:0709.4364.

[22] Chris Heunen, Nicolaas P. Landsman \& Bas Spitters (2011): Bohrification. In Hans Halvorson, editor: Deep Beauty, Cambridge University Press, pp. 271-313, doi:10.1017/CBO9780511976971.008. ArXiv:0909.3468.

[23] Chris Heunen, Nicolaas P. Landsman \& Bas Spitters (2012): Bohrification of operator algebras and quantum logic. Synthese 186, pp. 719-752, doi 10.1007/s11229-011-9918-4

[24] J.M.E. Hyland (1981): Function Spaces in the Category of Locales. In B. Banaschewski \& R.-E. Hoffmann, editors: Continuous Lattices: Proceedings, Bremen, 1979, Lecture Notes in Mathematics 871, SpringerVerlag, pp. 264-281, doi:10.1007/BFb0089910.

[25] Chris J. Isham \& Jeremy Butterfield (1998): A topos perspective on the Kochen-Specker theorem: I. Quantum States as Generalized Valuations. International Journal of Theoretical Physics 37(11), pp. 2669-2733, doi $10.1023 / \mathrm{A}: 1026680806775$.

[26] I.M. James (1989): Fibrewise Topology. Cambridge Tracts in Mathematics 91, Cambridge University Press, Cambridge, doi $10.1017 /$ CBO9780511896835 
[27] Peter T. Johnstone (1984): Open locales and exponentiation. In J.W. Gray, editor: Mathematical Applications of Category Theory, Contemporary Mathematics 30, American Mathematical Society, pp. 84-116, doi $10.1090 / \mathrm{conm} / 030 / 749770$

[28] Peter T. Johnstone \& André Joyal (1982): Continuous categories and exponentiable toposes. Journal of Pure and Applied Algebra 25(3), pp. 255-296, doi:10.1016/0022-4049(82)90083-4

[29] P.T. Johnstone (1982): Stone Spaces. Cambridge Studies in Advanced Mathematics 3, Cambridge University Press.

[30] P.T. Johnstone (2002): Sketches of an Elephant: A Topos Theory Compendium, vol. 1. Oxford Logic Guides 44, Oxford University Press.

[31] P.T. Johnstone (2002): Sketches of an Elephant: A Topos Theory Compendium, vol. 2. Oxford Logic Guides 44, Oxford University Press.

[32] André Joyal \& Miles Tierney (1984): An extension of the Galois theory of Grothendieck. Memoirs of the American Mathematical Society 51(309), doi $10.1090 / \mathrm{memo} / 0309$

[33] Saunders Mac Lane \& Ieke Moerdijk (1992): Sheaves in Geometry and Logic. Springer, doi 10.1007/978-14612-0927-0.

[34] I. Moerdijk (1986): Continuous Fibrations and Inverse Limits of Toposes. Compositio Mathematica 58, pp. 45-72. Available at http://www.numdam.org/item?id=CM_1986_-58_1_45_0

[35] Joost Nuiten (2011): Bohrification of local nets of observables. Ph.D. thesis, Utrecht. Available at http: //arxiv.org/abs/1109.1397.

[36] Erik Palmgren \& Steven Vickers (2007): Partial Horn Logic and Cartesian Categories. Annals of Pure and Applied Logic 145(3), pp. 314-353, doi:10.1016/j.apal.2006.10.001.

[37] Bas Spitters (2012): The Space of Measurement Outcomes as a Spectral Invariant for Non-Commutative Algebras. Foundations of Physics 42, pp. 896-908, doi 10.1007/s10701-011-9619-3.

[38] S.J. Vickers \& C.F. Townsend (2004): A Universal Characterization of the Double Powerlocale. Theoretical Computer Science 316, pp. 297-321, doi $10.1016 /$ j.tcs.2004.01.034

[39] Steven Vickers (1989): Topology via Logic. Cambridge University Press.

[40] Steven Vickers (1993): Information Systems for Continuous Posets. Theoretical Computer Science 114, pp. 201-229, doi:10.1016/0304-3975(93)90072-2.

[41] Steven Vickers (2004): The Double Powerlocale and Exponentiation: A Case Study in Geometric Reasoning. Theory and Applications of Categories 12, pp. 372-422. Available at http://www.tac.mta.ca/tac/ index.html\#vol12.

[42] Steven Vickers (2004): Entailment Systems for Stably Locally Compact Locales. Theoretical Computer Science 316, pp. 259-296, doi: 10.1016/j.tcs.2004.01.033.

[43] Steven Vickers (2005): Localic Completion of Generalized Metric Spaces I. Theory and Applications of Categories 14, pp. 328-356. Available at http://www.tac.mta.ca/tac/index.html\#vol14

[44] Steven Vickers (2006): Compactness in Locales and in Formal Topology. Annals of Pure and Applied Logic 137, pp. 413-438, doi:10.1016/j.apal.2005.05.028.

[45] Steven Vickers (2007): Locales and Toposes as Spaces. In Marco Aiello, Ian E. Pratt-Hartmann \& Johan F.A.K. van Benthem, editors: Handbook of Spatial Logics, chapter 8, Springer, pp. 429-496, doi $10.1007 / 978-1-4020-5587-4 \_8$.

[46] Steven Vickers (2009): Localic Completion of Generalized Metric Spaces II: Powerlocales. Journal of Logic and Analysis 1(11), pp. 1-48, doi:10.4115/jla.2009.1.11.

[47] Steven Vickers (2011): A Monad of Valuation Locales. Available at http://www.cs.bham.ac.uk/ sjv/ Riesz.pdf.

[48] Steven Vickers (2014): Continuity and Geometric Logic. Journal of Applied Logic 12(1), pp. 14-27, doi: $10.1016 /$ j.jal.2013.07.004. 
[49] K. Viglas (2004): Topos Aspects of the Extended Priestley Duality. Ph.D. thesis, Department of Computing, Imperial College, London.

[50] Sander Wolters (2013): A Comparison of Two Topos-Theoretic Approaches to Quantum Theory. Communications in Mathematical Physics 317(1), pp. 3-53, doi 10.1007/s00220-012-1652-3. ArXiv:1010.2031.

[51] Sander A.M. Wolters \& Hans Halvorson (2013): Independence Conditions for Nets of Local Algebras as Sheaf Conditions. Available at http://arxiv.org/abs/1309.5639. 